ARTICLE

\title{
Human Tra2 proteins jointly control a CHEK1 splicing switch among alternative and constitutive target exons
}

Andrew Best ${ }^{1}$, Katherine James ${ }^{2}$, Caroline Dalgliesh', Elaine Hong ${ }^{3}$, Mahsa Kheirolahi-Kouhestani ${ }^{1}$, Tomaz Curk4 , Yaobo Xu1, Marina Danilenko', Rafiq Hussain¹, Bernard Keavney ${ }^{1,5}$, Anil Wipat ${ }^{2}$, Roscoe Klinck ${ }^{6}$, Ian G. Cowell7, Ka Cheong Lee ${ }^{7}$, Caroline A. Austin ${ }^{7}$, Julian P. Venables ${ }^{7}$, Benoit Chabot $^{6}$, Mauro Santibanez Koref', Alison Tyson-Capper ${ }^{3} \&$ David J. Elliott ${ }^{1}$

\begin{abstract}
Alternative splicing-the production of multiple messenger RNA isoforms from a single gene-is regulated in part by RNA binding proteins. While the RBPs transformer2 alpha (Tra2 $\alpha$ ) and Tra2 $\beta$ have both been implicated in the regulation of alternative splicing, their relative contributions to this process are not well understood. Here we find simultaneousbut not individual-depletion of $\operatorname{Tra} 2 \alpha$ and Tra2 $\beta$ induces substantial shifts in splicing of endogenous Tra2 $\beta$ target exons, and that both constitutive and alternative target exons are under dual Tra2 $\alpha$-Tra2 $\beta$ control. Target exons are enriched in genes associated with chromosome biology including CHEK1, which encodes a key DNA damage response protein. Dual Tra2 protein depletion reduces expression of full-length $\mathrm{CHK1}$ protein, results in the accumulation of the DNA damage marker $\gamma \mathrm{H} 2 \mathrm{AX}$ and decreased cell viability. We conclude Tra2 proteins jointly control constitutive and alternative splicing patterns via paralog compensation to control pathways essential to the maintenance of cell viability.
\end{abstract}

\footnotetext{
${ }^{1}$ Institute of Genetic Medicine, Newcastle University, Central Parkway, Newcastle NE1 3BZ, UK. ${ }^{2}$ School of Computing Science, Claremont Tower, Newcastle University, Newcastle upon Tyne NE1 7RU, UK. ${ }^{3}$ Institute for Cellular Medicine, Newcastle University, Framlington Place, Newcastle NE2 4HH, UK. ${ }^{4}$ Faculty of Computer and Information Science, University of Ljubljana, Trzaska cesta 25, SI-1000, Ljubljana, Slovenia. ${ }^{5}$ Institute of Cardiovascular Sciences, The University of Manchester, Manchester M13 9NT, UK. ${ }^{6}$ Department of Microbiology and Infectious Diseases, Faculty of Medicine and Health Sciences, Université de Sherbrooke, Sherbrooke, Québec, Canada J1E 4K8. ${ }^{7}$ Institute for Cell and Molecular Biosciences, Newcastle University, Newcastle NE2 4HH, UK. Correspondence and requests for materials should be addressed to D.J.E. (email: David.Elliott@ncl.ac.uk)
} 
uman genes encode long precursor messenger RNAs (mRNAs) that are extensively processed before nuclear export. This maturation includes the splicing of exons, which normally occurs with high fidelity to create functional mRNAs ${ }^{1}$. Constitutive exons splice into all mRNAs transcribed from a gene, while alternative exons are sometimes included and sometimes skipped ${ }^{2}$. Human protein-coding genes each produce an average of three mRNA isoforms through alternative splicing, many of which are differentially regulated ${ }^{3}$. RNA binding proteins play a key role in transforming precursor RNAs into mRNAs. Although RNA-binding proteins can regulate many transcripts in parallel, some splicing regulatory proteins preferentially engage with transcripts belonging to specific functional classes, including Nova proteins (synapse functions), Fox proteins (neuromuscular, cytoskeleton and EMT functions), PTB proteins (cytoskeleton functions) and T-STAR (synapse functions $)^{4-8}$.

Transformer2 (Tra2) proteins are involved in splicing control $^{9,10}$. First discovered in insects, Tra2 proteins form an essential component of the alternative splicing complex that controls fly sexual differentiation ${ }^{11,12}$. Tra2 proteins are conserved across the animal kingdom, but separate gene paralogs encoding Tra2 $\alpha$ and Tra2 $\beta$ proteins evolved early in vertebrate evolution ${ }^{13,14}$. Knockout experiments in mice show that Tra2 $\beta$ is essential for embryonic and brain development ${ }^{15-18}$. In humans, Tra2 $\beta$ expression levels change in several cancers (reviewed by Best et $a .^{19}$ ), and Tra2 $\beta$ is implicated in the pathology of other diseases including spinal muscular atrophy ${ }^{20}$, Alzheimer's disease ${ }^{21}$ and frontotemporal dementia and Parkinsonism linked to chromosome 17 (ref. 22).

Tra2 proteins have amino- and carboxy-terminal domains enriched in arginine and serine residues (RS domains) flanking a single central RNA recognition motif (RRM) and so resemble the relatively well characterized core group of 12 SR proteins that control both constitutive and alternative splicing as well as other aspects of RNA metabolism ${ }^{23-25}$. Each core SR protein contains $\mathrm{N}$-terminal RRMs and single C-terminal RS domains. However, unlike the core SR proteins all current data implicate Tra2 proteins solely in alternative splicing rather than constitutive splicing ${ }^{10,26}$, and only SR proteins and not Tra2 proteins can provide splicing activity to S100 extracts ${ }^{26}$.

To regulate splicing inclusion Tra $2 \beta$ binds to AGAA-rich and CAA-rich target RNA sequences. These RNA protein interactions have been resolved at the atomic level ${ }^{9,27}$. Endogenous Tra2 $\beta$ target RNAs have been identified using HITS-CLIP ${ }^{18}$, RIP-seq ${ }^{28}$, shRNA depletion ${ }^{29}$ and microarrays ${ }^{17}$, but important fundamental questions still remain as to the identity of the biological targets and the functions of vertebrate Tra 2 proteins. These include whether endogenous Tra2 $\alpha$ and Tra2 $\beta$ proteins jointly control the same splicing targets, and if so what these shared targets are? Although $\operatorname{Tr} 2 \alpha$ and $\operatorname{Tra} 2 \beta$ both activate splicing of the same model exons when overexpressed in transfected HEK-293 cells (suggesting redundant functions) ${ }^{18}$, the Tra2a gene alone is not sufficient to maintain viability in Tra $2 b$ knockout mice (suggesting specific functions) ${ }^{15}$. Another question relates to how $\operatorname{Tra} 2 \alpha$ and $\operatorname{Tra} 2 \beta$ interact with each other? We previously found that Tra $2 \beta$ protein binds to a poison exon in the TRA2A gene to activate poison exon inclusion ${ }^{18}$. Poison exons introduce premature translation termination codons into mRNAs so as to inhibit translation of full-length proteins and are often regulatory ${ }^{18,30-32}$, but whether Tra2 $\alpha$ might reciprocally control Tra $2 \beta$ expression is not known.

Here we address these questions in human MDA-MB-231 cells that model invasive breast cancer. We find asymmetric splicing feedback control pathways between Tra2 $\alpha$ and Tra2 $\beta$ that buffer splicing defects caused by depletion of either Tra $2 \alpha$ or Tra $2 \beta$ protein alone. Overriding these feedback control pathways by joint depletion of both Tra $2 \alpha$ and Tra $2 \beta$ globally identifies Tra2dependent target exons, and reveals critical roles for these proteins in DNA damage control and cell viability.

\section{Results}

Tra2 $\beta$ efficiently suppresses Tra2 $\alpha$ protein expression. To test for in vivo interactions between $\operatorname{Tra} 2 \alpha$ and $\operatorname{Tra} 2 \beta$ proteins, we monitored their expression levels using western blots. Consistent with predictions from our previous study ${ }^{18}$, Tra $2 \alpha$ protein levels were normally very low but significantly increased after small interfering RNA (siRNA)-mediated depletion of Tra2 $\beta$ (Fig. 1a top panel, compare lanes 1 and 3, and Fig. 1b). Although weak, the Tra $2 \alpha$ western blot signal was of the predicted size and was almost completely eliminated following transfection with a TRA2A-specific siRNA (Fig. 1a top panel, compare lanes 1 and 2). Tra $2 \alpha$ protein depletion had less effect on Tra2 $\beta$ protein levels (Fig. 1a, middle panel and Fig. 1b). Western blot analysis confirmed this effect for two independent sets of siRNAs targeted against different parts of the respective mRNAs (Supplementary Fig. 1).

Consistent with Tra2 $\beta$ protein repressing Tra $2 \alpha$ expression via poison exon activation, siRNA-mediated depletion of Tra $2 \beta$ led to strongly reduced splicing inclusion of the TRA2A poison exon (Fig. 1d, upper panel). siRNA-mediated depletion of $\operatorname{Tra} 2 \alpha$ protein led to a smaller but detectable effect on splicing inclusion of the TRA2B poison exon (Fig. 1d, lower panel). Analysis of TRA2A and TRA2B steady state mRNA expression levels by quantitative PCR confirmed that each protein also negatively regulates the expression of the other at the RNA level (Fig. 1c).

The TRA2A and TRA2B genes are differentially expressed. RNA-seq of MDA-MB-231 cells indicated that the TRA2B gene is expressed at much higher levels than the TRA2A gene (Fig. 1e shows one of three biological replicate RNA-seq analyses, with the height of the $y$ axis showing read depth and so indicating relative gene expression levels). This provides a potential mechanism for why Tra $2 \beta$ represses Tra $2 \alpha$ protein expression more than vice versa, since lower cellular concentrations of Tra $2 \alpha$ would be less able to activate splicing of the TRA2B poison exon.

We used iCLIP $^{33}$ to systematically map the transcriptome-wide binding sites of human Tra2 $\beta$ in MDA-MB-231 cells. Endogenous Tra2 $\beta$ protein was efficiently immunoprecipitated along with radiolabelled crosslinked RNA. A single radiolabelled RNA protein adduct of $\sim 40 \mathrm{kDa}$ was identified at high RNase concentrations, just above the known molecular weight of uncrosslinked endogenous Tra $2 \beta$ protein $(37 \mathrm{kDa})$ (arrowed in Supplementary Fig. 2a). Lower RNase concentrations enabled endogenous Tra2 $\beta$ binding sites to be mapped across the MDAMB-231 cell transcriptome in biological triplicate iCLIP experiments. Following deep sequencing, 7,443,903 reads were successfully mapped back to the human genome, of which $3,338,710$ were unique cDNA reads used for downstream analysis (Supplementary Data 1). These individual sequencing reads are subsequently referred to as iCLIP tags. The only clusters of Tra $2 \beta$ iCLIP tags, which mapped to the human TRA2B and TRA2A genes from all three biological replicates were within their respective poison exons (Fig. 1e). Despite much lower levels of overall TRA2A gene expression, the TRA2A poison exon had a similar number of Tra2 $\beta$ iCLIP tags as the TRA $2 B$ poison exon. This suggests the TRA2A poison exon is a stronger physiological target for Tra $2 \beta$ binding than the TRA2B poison exon (the TRA $2 A$ poison exon also has a much higher density of AGAA Tra2 $\beta$ binding sites than the TRA2B poison exon ${ }^{18}$ ). 
a

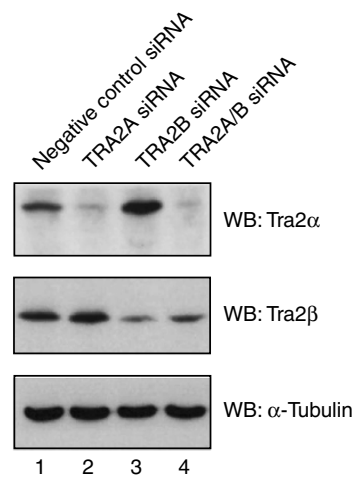

b
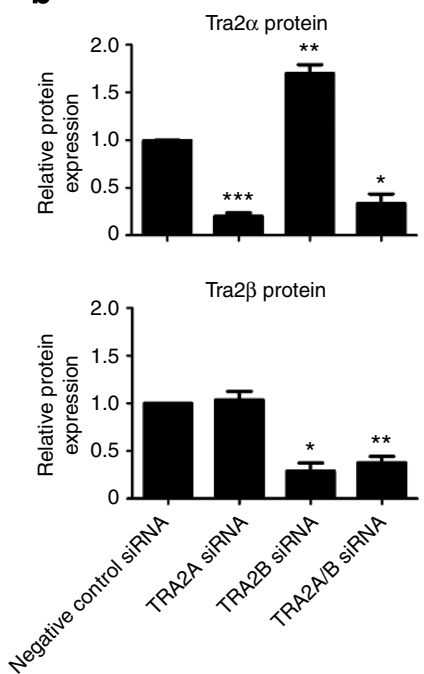

C

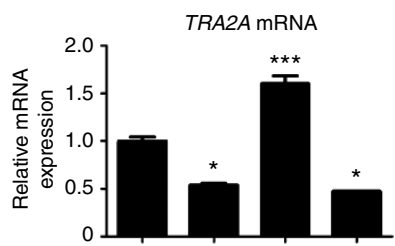

TRA2B mRNA

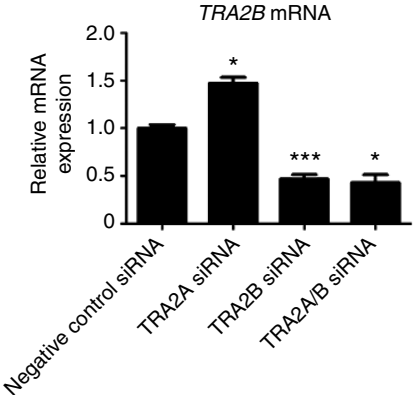

d
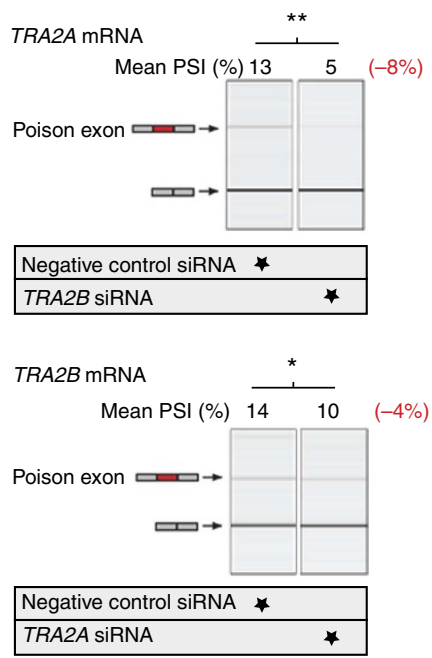

e
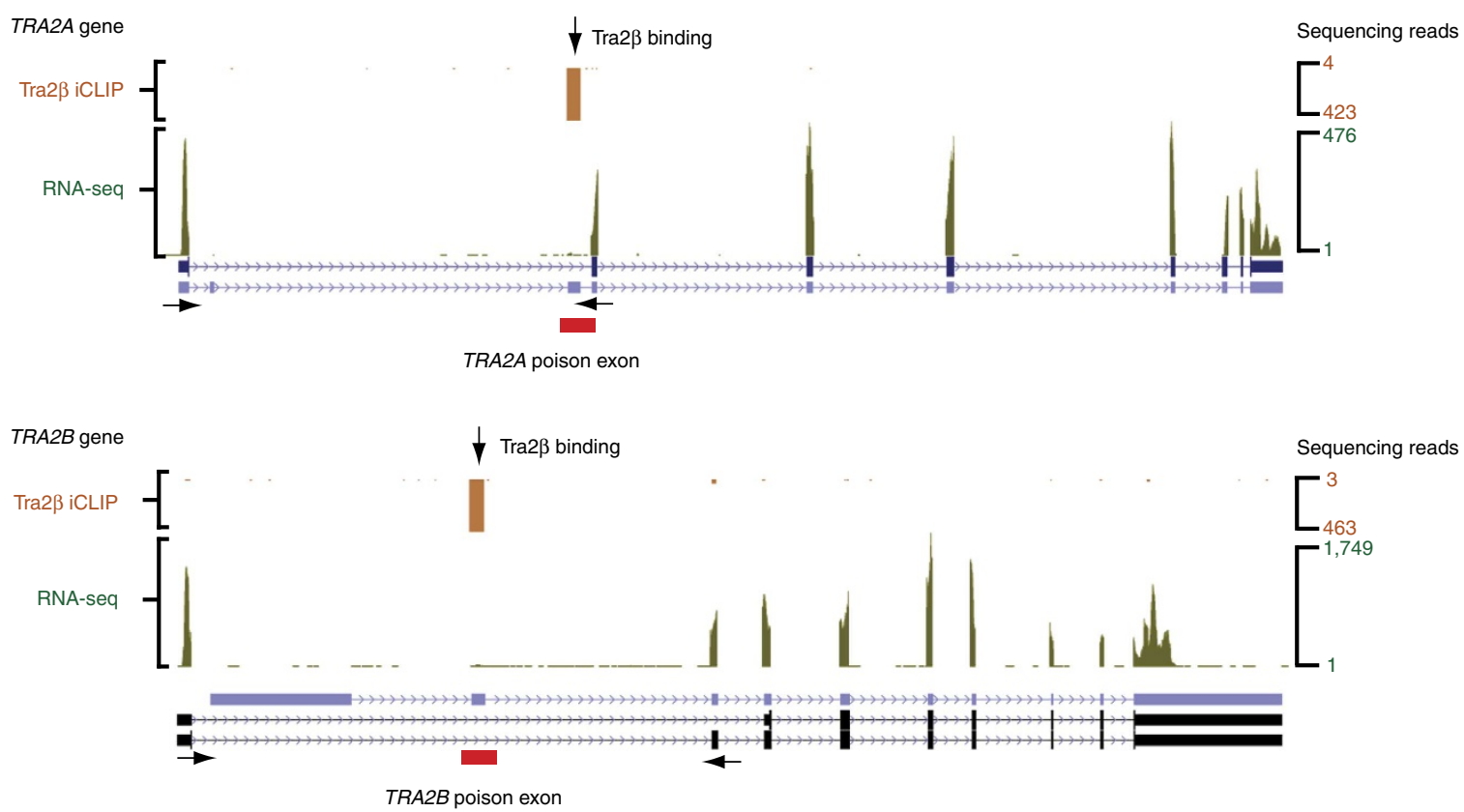

Figure 1 | Tra2 $\beta$ regulates Tra2 $\alpha$ protein expression. (a) Western blot analysis showing depletion of TRA2B induces reciprocal upregulation of Tra2 $\alpha$ protein expression, whereas depletion of TRA2A had minimal effect on Tra2 $\beta$ protein expression. (b) Quantitation of cross-regulation between Tra2 $\alpha$ and $\operatorname{Tr} 2 \beta$ at the protein level (Tra2 $\alpha$ and Tra2 $\beta$ protein expression were quantified relative to $\alpha$-Tubulin from three western blots using independent biological replicates). (c) Quantitation of cross-regulation between Tra2 $\alpha$ and Tra2 $\beta$ at the RNA level from quantitative PCR analysis of three independent biological replicates in MDA-MB-231 cells. (d) Splicing inclusion of the TRA2A poison exon is strongly reduced by depletion of endogenous Tra2 $\beta$ protein, whereas splicing inclusion of the TRA2B poison exon is less affected by depletion of Tra2 $\alpha$ protein. Splicing patterns were monitored by RT-PCR between flanking exons (arrowed) followed by capillary electrophoresis. (e) Screenshot from the UCSC genome browser ${ }^{35}$ showing the TRA2B and TRA2A genes, and the positions of aligned RNA-seq reads (green peaks) and Tra2 $\beta$ binding (orange clusters of significant cross-linking by Tra2 $\beta$ protein identified by biological triplicate iCLIP experiments) in MDA-MB-231 cells. Probability $(P)$ values were calculated using an independent two-sample $t$-test between negative control siRNA-treated cells and the gene-specific siRNA-treated cells (statistical significance shown as: ${ }^{\star} P<0.05,{ }^{\star \star} P<0.01,{ }^{\star \star \star} P<0.0001$ ). All data represented by bar charts was generated from three biological replicates and error bars represent the s.e.m.

Endogenous Tra2 $\alpha$ functionally compensates for loss of Tra2 $\beta$. The most frequently enriched pentamers recovered in the iCLIP tags were highly enriched in AGAA nucleotide sequences (Supplementary Fig. $2 b$ ), which is the Tra $2 \beta$ binding site predicted by HITS-CLIP for endogenous mouse Tra2 $\beta$, RIP-seq and from SELEX experiments using purified Tra $2 \beta$ proteins ${ }^{18,26,28}$. However, our combined human iCLIP data in MDA-MB-231 cells provided substantially more coverage than previously obtained in mouse testis ${ }^{18}$ (in which just 177,457 reads were mapped back to the mouse genome). In total, 1,546,290 (44.8\%) of unique cDNAs mapped to intronic regions, suggesting the Tra2 $\beta$ iCLIP experiment largely captured Tra2 $\beta$ interactions with pre-mRNAs. However, a further 1,169,374 (33.8\%) of unique cDNAs mapped to exons (5'UTR, $3^{\prime} \mathrm{UTR}$ or ORF), despite exons comprising only $\sim 1 \%$ of the genome. After correcting for the relative size of each genomic region (by dividing the 
number of unique cDNAs mapping to each genomic region by the relative size of that region within the genome), we find that Tra2 $\beta$ binding is highly enriched within exons: $76.8 \%$ of Tra2 $\beta$ iCLIP tags mapped to exons ( $5^{\prime}$ UTR, $3^{\prime}$ UTR or ORF), while a further $20.4 \%$ mapped to non-coding RNAs (Supplementary Fig. 2c).

We used our iCLIP data to screen for endogenous exons controlled by human Tra2 $\beta$. Alternative exon junctions $(+/-300 \mathrm{bp}$ of either splice site) were stratified to find those with the highest number of iCLIP tags relative to overall iCLIP coverage within the same gene. To test whether upregulation of Tra $2 \alpha$ protein expression could functionally compensate in splicing regulation for depletion of $\operatorname{Tra} 2 \beta$, we then monitored percentage splicing inclusion (PSI) of associated exons after single depletion of either Tra $2 \alpha$ or Tra $2 \beta$, or after combined depletion of both Tra $2 \alpha$ and Tra $2 \beta$ proteins.

Clusters of Tra2 $\beta$ iCLIP tags mapped to alternative exons in the ATRX, GLYR1 and CEP95 genes (Fig. 2a). Single depletion of either Tra $2 \alpha$ or Tra $2 \beta$ had only a small effect on the endogenous splicing pattern of these three exons, but joint depletion of both Tra $2 \alpha$ and Tra2 $\beta$ substantially decreased their splicing inclusion
(Fig. 2b). We obtained similar results with 14/14 Tra2 $\beta$ target exons identified by iCLIP analysis (Fig. 2c. Individual data for each of these tested exons are shown in Supplementary Fig. 3). In fact, of these 14 tested exons, some less-responsive exons including cassette exons within $P A M$ and $B D P 1$ only responded to depletion of both Tra2 proteins, and not to single depletion of Tra2 $\beta$ at all (Supplementary Fig. 3).

These data are consistent with maintenance of splicing patterns via paralog compensation, that is, following depletion of Tra2 $\beta$, upregulated Tra $2 \alpha$ is able to functionally substitute for Tra $2 \beta$ and largely maintain Tra 2 target exon inclusion. The Tra2 $\beta$ target exons inhibited more substantially by joint Tra 2 protein depletion compared with single depletion of either Tra2 $\alpha$ or Tra $2 \beta$ included SMN2 exon 7 (Supplementary Fig. 3), which is a candidate target for gene therapy in spinal muscular atrophy ${ }^{20}$.

Tra $2 \alpha$ and Tra2 $\beta$ control constitutive exon splicing patterns. Splicing profiles of candidate Tra2 $\beta$ target exons (containing Tra2 $\beta$ iCLIP tag coverage) were next analysed using RNA-seq after joint depletion of Tra $2 \alpha$ and Tra2 $\beta$ proteins, and changes

a

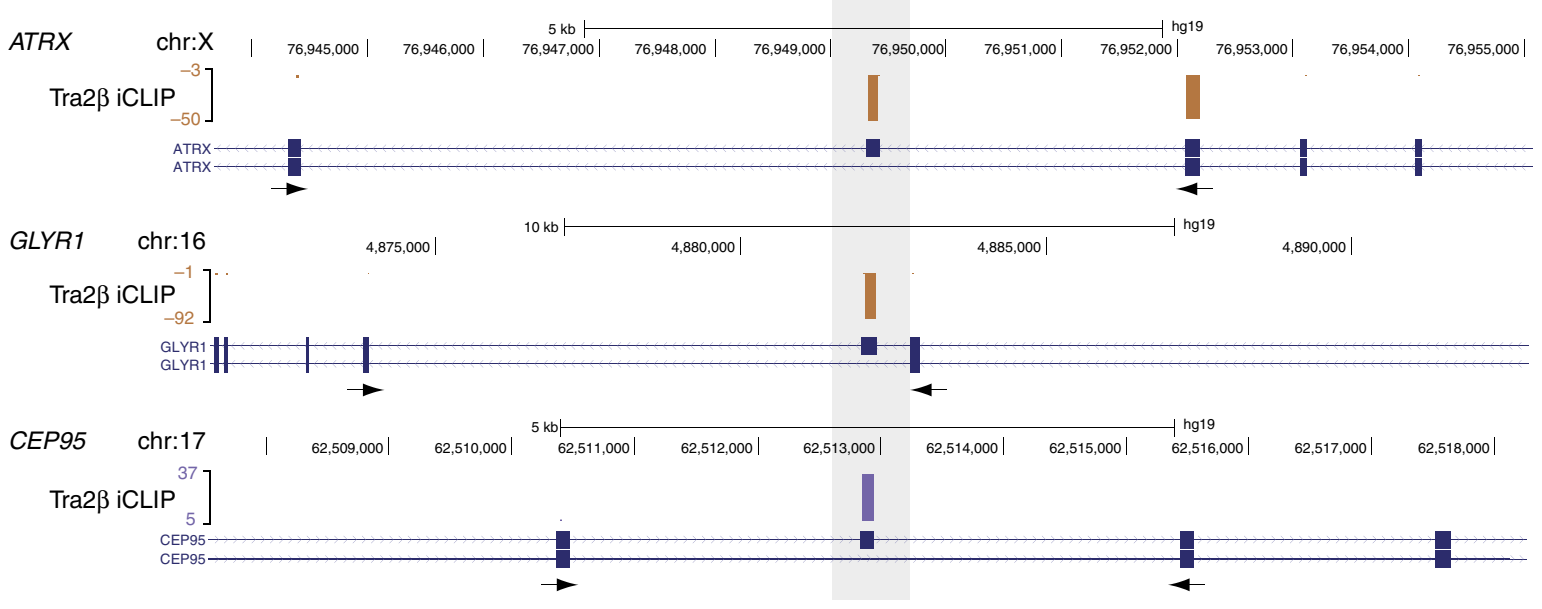

b

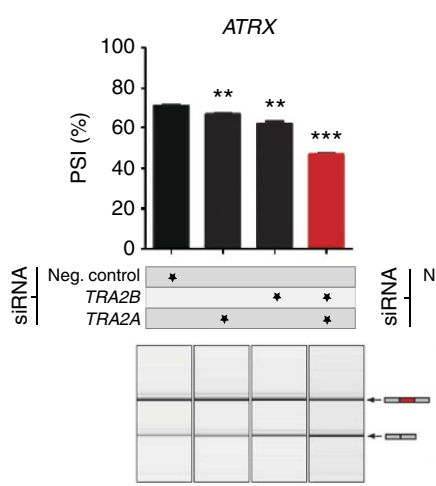

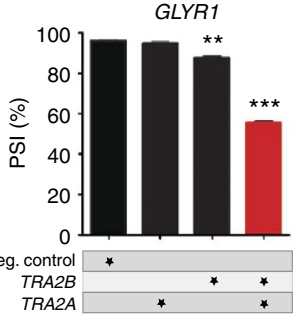

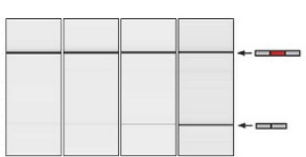

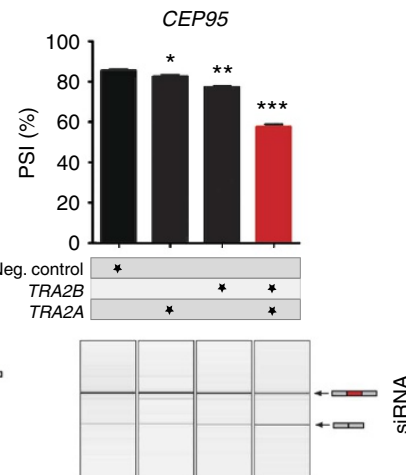

Figure 2 | Endogenous Tra2 $\alpha$ functionally compensates for loss of Tra2 $\beta$. (a) UCSC genome browser screenshot ${ }^{35}$ showing significant clusters of iCLIP tags mapping directly to alternatively spliced exons within the CEP95, GLYR1 and ATRX genes (position of target alternative exons highlighted in grey). (b) Splicing inclusion of novel Tra2 $\beta$ target exons within ATRX, GLYR1 and CEP95 were only slightly affected by depletion of either endogenous Tra2 $\alpha$ or $\operatorname{Tra} 2 \beta$ proteins, but were strongly affected by joint depletion of both Tra2 $\alpha$ and Tra2 $\beta$ (red). PSI levels were measured by RT-PCR and capillary gel electrophoresis (lower panels) in three biological replicates (upper panels). (c) Splicing inclusion of 14 novel Tra2 $\beta$ target exons showed minimal splicing response to single depletion of either Tra2 $\alpha$ or Tra2 $\beta$, but showed highly significant splicing changes after joint depletion of both Tra2 proteins (complete data for all 14 exons is provided in Supplementary Fig. 4). Probability $(P)$ values were calculated using an independent two-sample $t$-test between PSI levels of negative control siRNA-treated cells and TRA2A/TRA2B siRNA-treated cells (statistical significance: ${ }^{\star} P<0.05,{ }^{\star \star} P<0.01,{ }^{\star \star \star} P<0.0001$ ). All data represented by bar charts was generated from three biological replicates where error bars represent the s.e.m. 


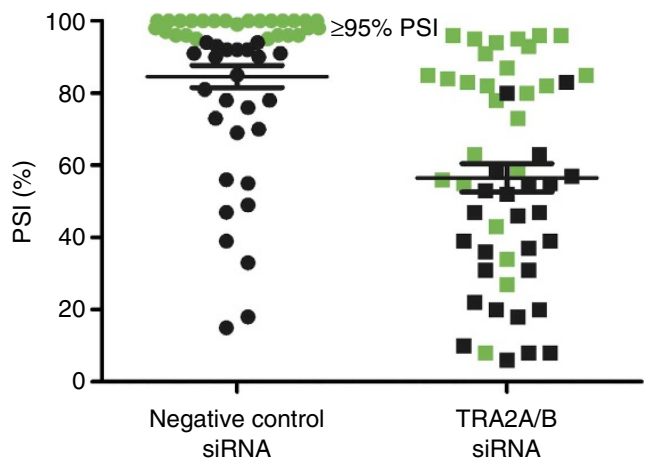

b

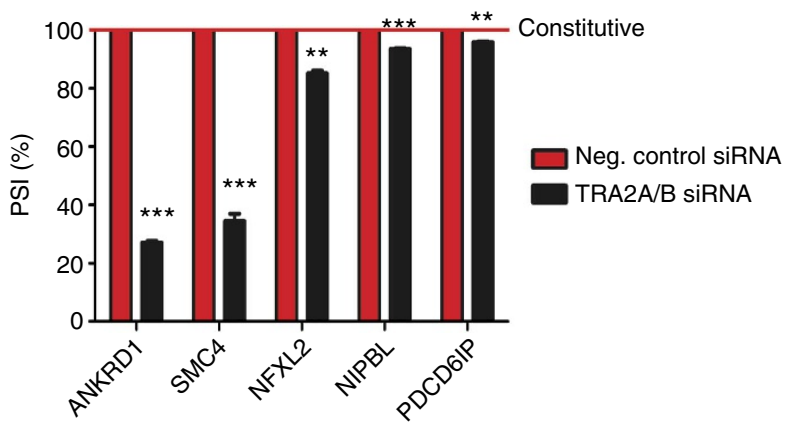

C
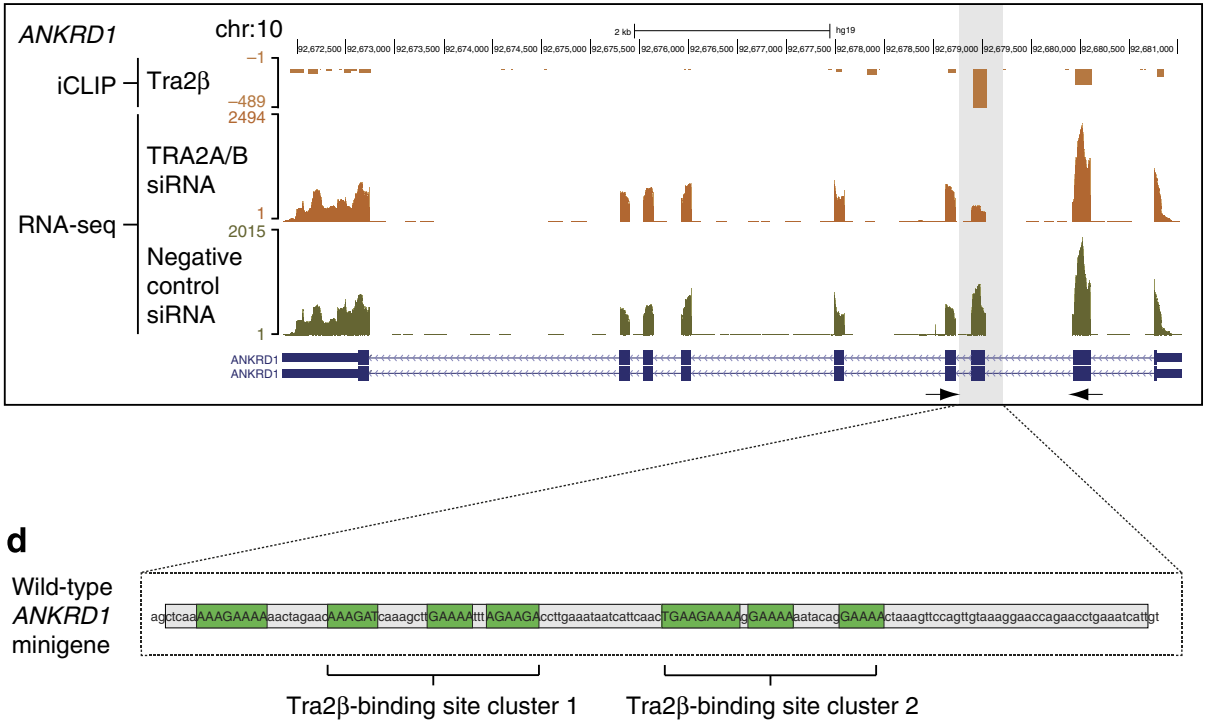

Mutation 1

Mutation 2 $\times \quad \times x$

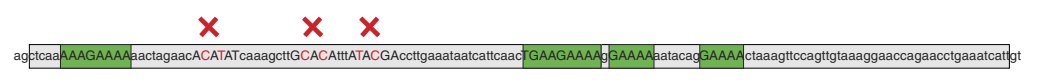

$\times \times x$
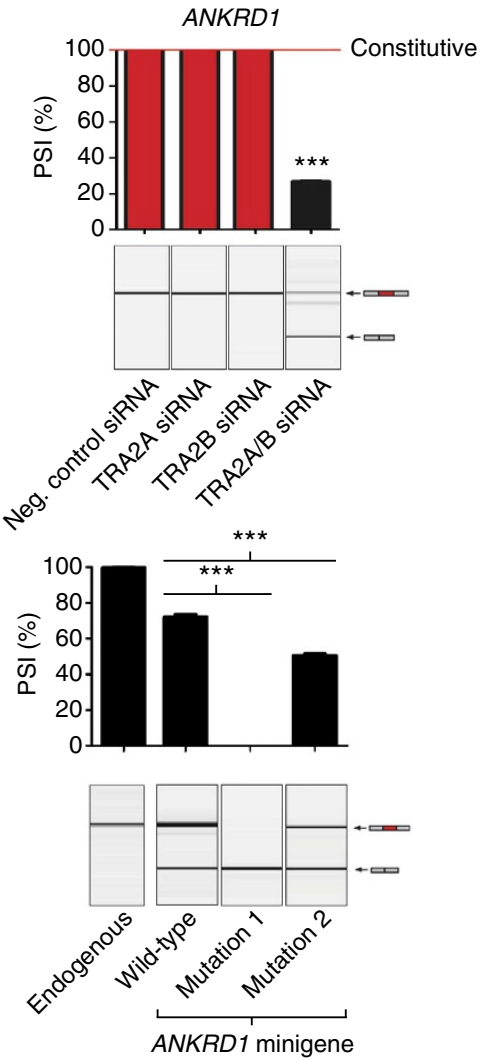

Figure 3 | Tra2 proteins control splicing of constitutively spliced target exons. (a) Many novel Tra2 $\alpha / \beta$-responsive exons normally have high levels of splicing inclusion in MDA-MB-231 cells. PSI levels of target exons are shown from negative control siRNA-treated cells and after joint Tra2 protein depletion. Exons included at equal to or greater than 95\% PSI in control MDA-MB-231 cells are highlighted in green. (b) The inclusion of five constitutively spliced Tra2 $\beta$ target exons is reduced after joint Tra2 $\alpha / \operatorname{Tra} 2 \beta$ protein depletion. PSI levels were measured by RT-PCR and capillary gel electrophoresis. (c) The splicing profile of the ANKRD1 gene changes in response to joint depletion of Tra2 proteins. Combined iCLIP and RNA-seq data were visualized on the UCSC genome browser ${ }^{35}$ (left panel), and splicing inclusion levels directly measured using RT-PCR (right panel). Probability ( $P$ ) values were calculated using an independent two-sample $t$-test between the PSI levels of negative control siRNA-treated cells and the gene-specific siRNA-treated cells (statistical significance: $\left.{ }^{\star} P<0.05,{ }^{\star \star} P<0.01,{ }^{\star \star \star} P<0.0001\right)$. All data represented by bar charts was generated from three biological replicates and error bars represent s.e.m. (d) Tra2 $\beta$ binding sites in the ANKRD1 exon are essential for its splicing inclusion. The wild-type minigene contained two clusters of Tra2 $\beta$ binding sites, which were independently altered by mutagenesis (left panel). The PSI of the resulting exons was measured after transfection into HEK-293 cells at endogenous Tra2 $\beta$ protein concentrations (right panel). Probability $(P)$ values were calculated using an independent two-sample $t$-test between PSI levels of the wild-type and mutated versions of the ANKRD1 minigene (statistical significance: ${ }^{\star} P<0.05,{ }^{\star \star} P<0.01,{ }^{\star \star \star} P<0.0001$ ). All data represented by bar charts was generated from three biological replicates and error bars represent the s.e.m.

validated by reverse transcriptase (reverse transcriptase-PCR). From the initial panel of 30 Tra2 protein-responsive alternative exons that we identified, $7 / 30$ were included at $100 \%$ and $17 / 30$ had a PSI greater or equal to 95\% in MDA-MB-231 cells (Fig. 3a). These results suggested that Tra2 proteins might be important for the inclusion of constitutive exons (in addition to their expected function in alternative exon splicing regulation), or alternatively that Tra2 protein expression levels in MDA-MB-231 cells are sufficient to promote $100 \%$ inclusion of some alternative exons.

To distinguish between these possibilities, we used our iCLIP and RNA-seq data to search for splicing changes in exons that have never previously been annotated as alternatively spliced in human cells ${ }^{34,35}$. Such exons were found in the ANKRD1, SMC4, NFXL2, NIPBL and PDCD6IP genes. Each of these five exons were spliced at $100 \%$ PSI in MDA-MB-231 cells but skipped at 
different levels after Tra2 protein depletion (Fig. 3b). The constitutively spliced exon within ANKRD1 showed the largest change (Fig. $3 c$ ), a $-73 \%$ point switch in PSI after joint depletion of Tra2 $\alpha$ and Tra2 $\beta$. To further dissect splicing control, we cloned the $A N K R D 1$ exon and its flanking intronic sequences between $\beta$-globin exons in a minigene construct. Transfection experiments showed the ANKRD1 exon is included at $72 \%$ PSI when expressed from this minigene, indicating it lacks some important sequences for splicing (the endogenous ANKRD1 exon was $100 \%$ included in these transfected HEK-293 cells, Fig. 3d). Two clusters of GAA-rich Tra2 $\beta$ binding sites were present in the $A N K R D 1$ exon (Tra2 $\beta$ binding site clusters 1 and 2 ). Mutation of either Tra2 $\beta$ binding site cluster negatively impacted splicing inclusion, showing an essential role for these binding sites in modulating the inclusion of the ANKRD1 exon. In particular, mutation of Tra2 $\beta$ binding site cluster 1 (to create Mutant M1) completely abolished splicing inclusion of the ANKRD1 constitutive exon (Fig. 3d, right panel).

In total, we identified and validated 53 human splicing targets, which were both directly bound and jointly controlled by Tra2 proteins, including both alternative and constitutive exons (Fig. 4a; Supplementary Fig. 10; Supplementary Data 2). As well as SMN2 exon 7 (ref. 20), these included the NASP-T exon and the TRA2A poison exon, orthologs of which have both been previously identified as functional Tra $2 \beta$ splicing targets in the mouse testis by HITS-CLIP ${ }^{18}$. The NASP-T exon was also in the data set of Tra2 $\beta$ targets identified by RIP-seq ${ }^{28}$. However, the vast majority of the dual Tra $2 \alpha / \operatorname{Tra} 2 \beta$ target exons identified here are novel. The PSI changes for individual genes in response to joint Tra $2 \alpha$ and Tra2 $\beta$ protein depletion ranged between -4 and $-92 \%$ points (measured by RT-PCR, Fig. 4a; Supplementary Data 2), indicating that individual Tra2-dependent exons have different intrinsic requirements for Tra2 proteins. The length of Tra2-dependent exons ranged from 64 nucleotides at the smallest (a cassette exon in the SMYD2 gene, which showed a $-5 \%$ point switch in PSI in response to Tra2 protein depletion), to 5916 nucleotides at the largest (an unusually large internal cassette exon in the SON gene, which showed a -8 point PSI change in response to endogenous Tra2 protein depletion) (Supplementary Data 2). A further 38 exons had Tra2 $\beta$ iCLIP tag coverage, but did not detectably respond to Tra2 protein depletion in MDAMB-231 cells: possibly this latter class of exons either might need a relatively small amount of Tra2 protein to be included, or alternatively they might not be controlled by Tra2 proteins in MDA-MB-231 cells (Supplementary Data 2). In a comparison between Tra2 responsive and non-responsive exons, the only statistically significant difference was the density of Tra2 $\beta$ binding sites in the more highly responsive exons (exons showing $>15$ PSI change following joint Tra $2 \alpha / \operatorname{Tra} 2 \beta$ depletion, Fig. 4b). High resolution iCLIP maps of the individual exons are shown in Supplementary Fig. 10.

Tra2 proteins are highly specific splicing regulators. To establish the relative role of Tra2 proteins in controlling the identified panel of target exons, we probed a custom plate containing cDNAs, where we had systematically knocked down 53 known splicing regulators in MDA-MB-231 cells ${ }^{5}$ (Fig. 4c). Strikingly, of all the knockdowns tested, only double knockdown of Tra2 proteins shifted ANKRD1 splicing (constitutive exon) and joint Tra2 depletion also had the largest effect on splicing for GLYR1 (alternative exon). An intermediate situation was observed for SMC4 (constitutive exon) in which knockdown of $S N R P 70$ (encoding U170K) also reduced splicing inclusion, as did knockdown of SRPK1. The splicing inclusion pattern of $C H E K 1$ was strongly shifted ( -78 point PSI switch) by joint depletion of Tra2 proteins, but consistent with broader mechanisms of combinatorial control, significant shifts were also seen after depletion of three core U2 snRNP components, which are thought to be important for splice site commitment for all exons. Depletion of other constitutive splicing factors such as SFRS2, hnRNPK, hnRNPC2, KHSRP and CDC5L also affected CHEK1 exon 3 splicing.

Since our panel of splicing factor knockdowns was not exhaustive, we cannot exclude all combinations of combinatorial control. However, our data are at least consistent with Tra2 proteins being among the most quantitatively important splicing regulators for their individual target exons.

Tra2 splicing targets associate with chromosome biology. Gene ontology (GO) enrichment analysis of the 53 human genes containing Tra2-dependent exons revealed an enrichment of five functionally similar biological processes (Fig. 5a). Eleven of the 53 genes were annotated to one or more of these processes, with ten of the eleven genes being annotated to the term 'chromosome organization'. There was significant overlap in annotation between this process and annotation to the conceptually related terms 'histone modification' and 'chromatin modification' (Fig. 5b).

The BioGRID database ${ }^{36}$ was used to retrieve a network of functional interaction data involving genes containing Tra2dependent exons. In addition to the eleven genes directly annotated to the five enriched GO processes in Fig. 5a, a further 23 of the 53 genes (43.4\%) that contain Tra2-dependent exons also have functional interactions with genes annotated to these terms (Supplementary Fig. 4, and summarized in Fig. 5c). Interestingly, although indirectly connected within the network via these annotated genes, none of the 53 genes containing validated Tra2-dependent exons directly interact with one another in the BioGRID database (Supplementary Fig. 4).

Tra2 proteins control splicing of a key checkpoint protein. Among the Tra2 target exons involved in chromosome biology was exon 3 of the CHEK1 gene, which encodes the serine/ threonine protein kinase CHK1 that is involved in checkpoint control in response to DNA damage. iCLIP analysis identified significant Tra $2 \beta$ binding over CHEK1 exon 3 (Fig. 6a), and we observed a -55 point PSI switch for this exon in MDA-MB-231 cells after joint Tra2 protein depletion (Fig. 6b). Joint depletions of Tra $2 \alpha$ and Tra $2 \beta$ proteins also indicate CHEK 1 exon 3 splicing is under similar control in multiple cell types including MCF7, PC3 and HeLa (Fig. 6b).

Tra2 $\beta$ iCLIP tags mapped throughout CHEK1 exon 3, but were particularly enriched towards the $3^{\prime}$ splice site (Fig. 7a). We confirmed $C H E K 1$ exon 3 is a direct target for Tra $\beta$ binding in vitro by electrophoretic mobility shift assays (EMSAs) using radiolabelled RNA probes corresponding to portions of the exon sequence (Fig. 7b). RNA probe A corresponds to the part of CHEK 1 exon 3 with the most Tra $2 \beta$ iCLIP tags, and also contains the most predicted binding sites for Tra2 $\beta$ (shaded green in Fig. $7 \mathrm{~B}$, right hand side). RNA probe A was very efficiently shifted by even the lowest tested concentrations ( $25 \mathrm{ng}$ ) of Tra $2 \beta$ protein. RNA probe $B$ did not bind Tra $2 \beta$ protein as tightly (around $200 \mathrm{ng}$ Tra $2 \beta$ protein was needed to see a comparable shift) and also contained fewer Tra2 $\beta$ binding sites and mapped iCLIP tags. A control RNA probe corresponding to the flanking intron sequence did not shift even at the highest concentrations of Tra2 $\beta$ protein (this intron sequence contained no predicted Tra2 $\beta$ binding sites).

We confirmed that $C H E K 1$ exon 3 is a direct target for Tra2 $\beta$ splicing regulation using a minigene construct in which $C H E K 1$ exon 3 is flanked by $\beta$-globin exons (Fig. 7c). After transfection of 
a

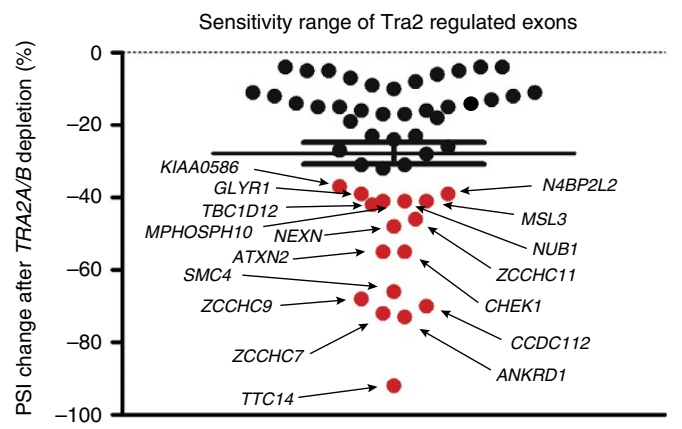

b

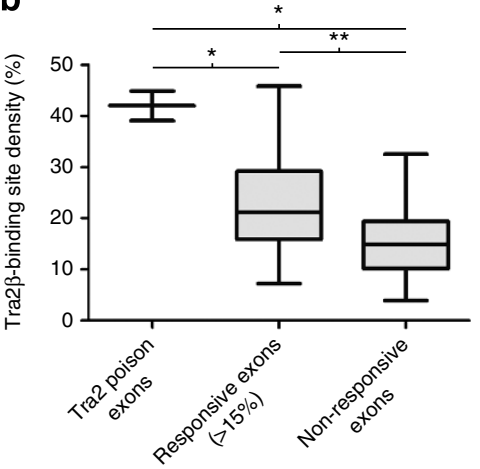

C
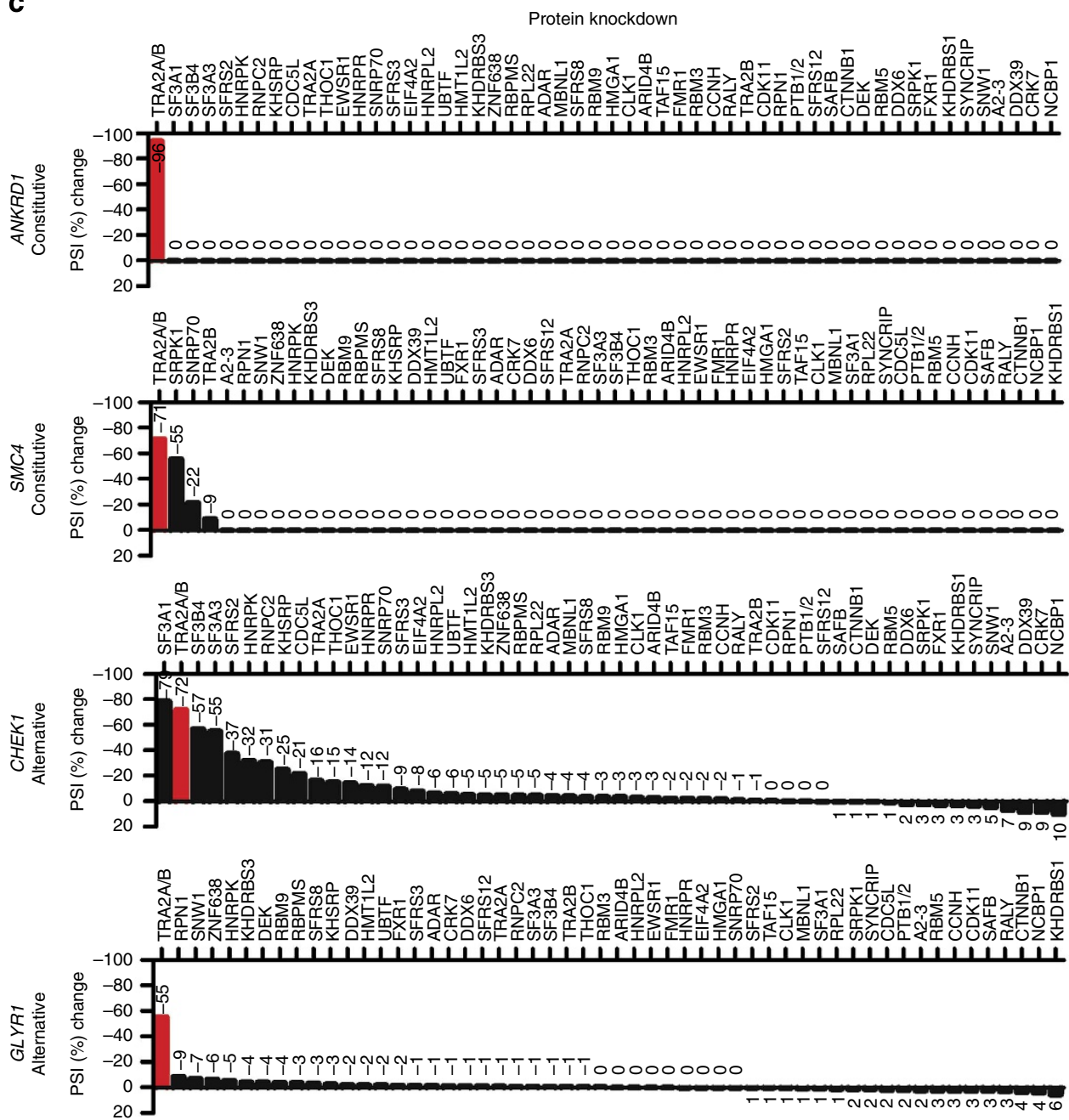

Figure 4 | Identification of exons jointly controlled by Tra2 $\alpha$ and Tra2 $\beta$ proteins. (a) Scatterplot showing amplitude of splicing response of 53 exons to joint depletion of endogenous Tra2 $\alpha$ and Tra2 $\beta$ in MDA-MB-231 cells. The genes corresponding to the highest amplitude PSI changes after joint Tra2 protein depletion are labelled and highlighted in red. (b) Analysis of Tra2 $\beta$ binding site density (measured as a percentage of exon content) within groups of Tra $2 \beta$ target exons identified by iCLIP. Tra2 $\beta$ binding site density comparisons are shown between the Tra2 $\alpha$ and Tra2 $\beta$ poison exons; all exons that showed a greater than $15 \%$ point PSI change following joint Tra $2 \alpha$ and Tra2 $\beta$ depletion; and in the exons that bound Tra2 $\beta$ based on iCLIP tag coverage but did not respond to Tra2 $\alpha$ and Tra2 $\beta$ depletion. Probability $(P)$ values were calculated using an independent two-sample $t$-test (statistical significance: ${ }^{\star} P<0.05,{ }^{\star \star} P<0.01,{ }^{\star \star}{ }^{*} P<0.0001$ ). (c) Regulation of ANKRD1, SMC4, GLYR1 and CHEK1 splice variants following knockdown of a panel of RNA binding proteins (RBPs) in MDA-MB-231 cells ${ }^{5}$. The $y$ axis shows PSI change after joint Tra2 $\alpha$ and Tra2 $\beta$ depletion, with a negative number indicating splicing repression in the absence of these proteins.

this minigene into HEK-293 cells, CHEK1 exon 3 was skipped, but its splicing inclusion was strongly induced in response to co-transfection with either Tra2 $\beta$-GFP or Tra2 $\alpha$-GFP. No CHEK1 exon 3 splicing activation was observed after co- transfection of either Tra2 $\beta \Delta$ RRM-GFP (lacking the RRM) or GFP alone. Furthermore, point mutations of the Tra2 $\beta$ binding sites within the exon (wild-type binding sites shaded green, mutations shaded red in Fig. $7 \mathrm{c}$ right hand side) completely 
a

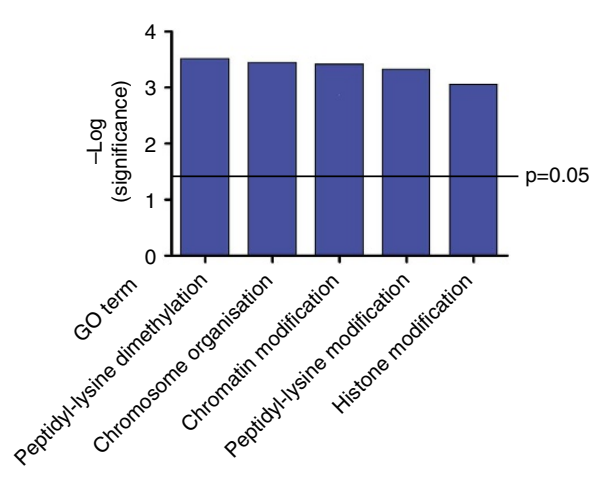

b

\section{GO term}

Peptidyl-lysine dimethylation Peptidyl-lysine modification Chromosome organization Histone modification Chromatin modification

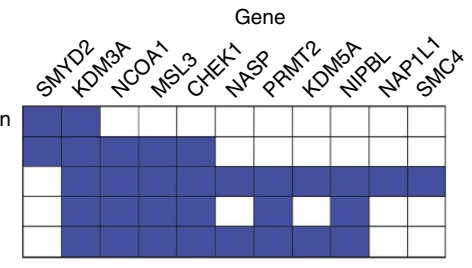

C

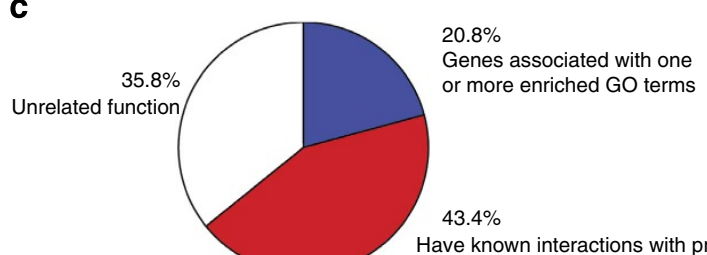

Have known interactions with proteins associated with one or more enriched GO terms

Figure 5 | Tra2 splicing targets are enriched in GO terms associated with chromosome biology. (a) GO enrichment analysis reveals splicing targets responsive to endogenous Tra2 $\alpha / \operatorname{Tra} 2 \beta$ protein concentrations are enriched in particular biological processes associated with chromosome biology.

(b) GO enrichment analysis showed some joint Tra2 $\alpha / \operatorname{Tra} 2 \beta$-responsive exons were annotated to multiple overlapping biological processes.

(c) Summary of GO and network analyses of joint Tra2 $\alpha$ and Tra2 $\beta$-dependent splicing targets. Individual segments of the pie chart show the percentage of $\operatorname{Tr} 2 \alpha / \operatorname{Tr} 2 \beta$ target genes directly annotated to the GO biological processes shown in part $\mathbf{a}$; the percentage of $\operatorname{Tra} 2 \alpha / \operatorname{Tra} 2 \beta$ target genes that interact within the BioGrid database with partners known to be involved in the biological processes shown in part $\mathbf{a}$; and the percentage of Tra $2 \alpha / \operatorname{Tra} 2 \beta$ target genes, which have unknown or unrelated functions. Full details of the BioGrid analysis are given in Supplementary Fig. 4.

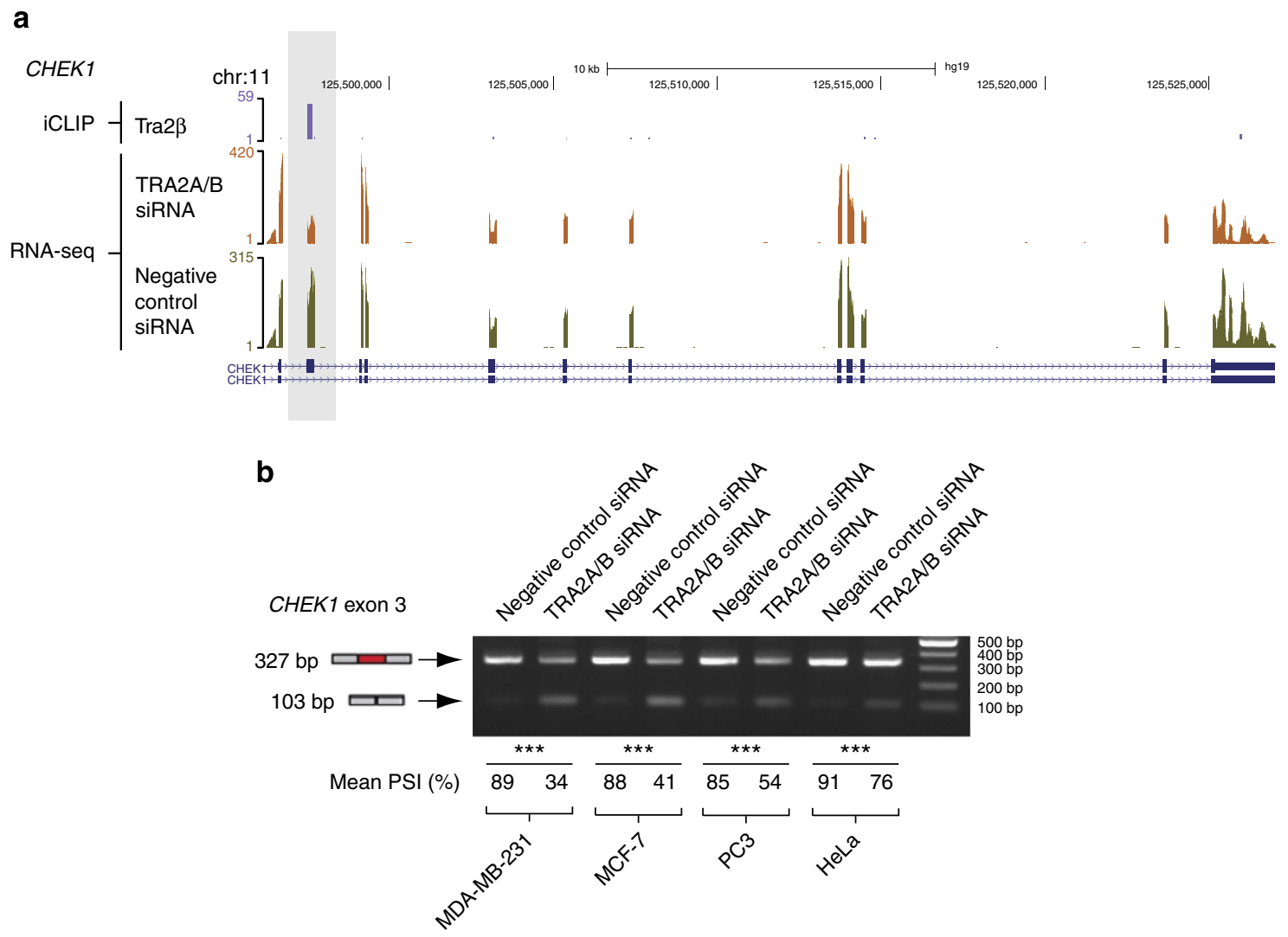

Figure 6 | Splicing inclusion of CHEK1 exon 3 decreases following joint depletion of Tra2 $\alpha$ and Tra2 $\beta$. (a) Screenshot ${ }^{35}$ from the UCSC genome browser showing the CHEK1 gene. Tra2 $\beta$ iCLIP tags are shown at the top in purple, and map predominantly to a known alternative exon (CHEK1 exon 3 ) that is skipped in one mRNA isoform (shaded grey). Example RNA-seq tracks from control MDA-MB-231 cells and after joint Tra2 $\alpha$ and Tra2 $\beta$ protein depletion are shown in green and orange respectively. (b) Splicing inclusion of CHEK1 exon 3 is inhibited by siRNA-mediated Tra2 $\alpha$ and Tra2 $\beta$ protein depletion in multiple cell types. Splicing inclusion was monitored by RT-PCR and agarose gel electrophoresis, and shown as a mean PSI value after analysis of three independent samples. Probability $(P)$ values were calculated using an independent two-sample $t$-test between PSI levels of negative control siRNA-treated cells and TRA2A/B siRNA-treated cells (statistical significance: ${ }^{\star} P<0.05,{ }^{\star \star} P<0.01,{ }^{\star \star \star} P<0.0001$ ). An uncropped gel is shown in Supplementary Fig. 13. 
a

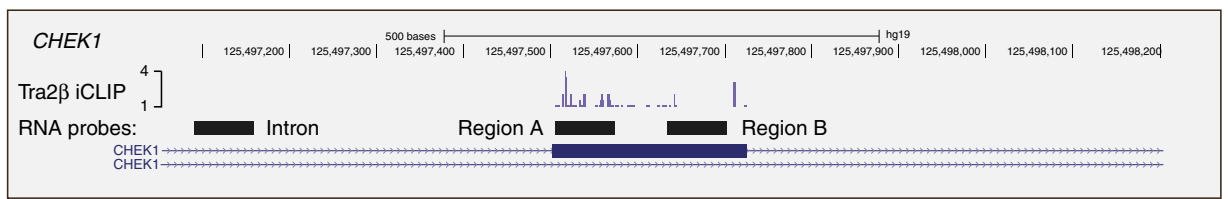

\section{b}
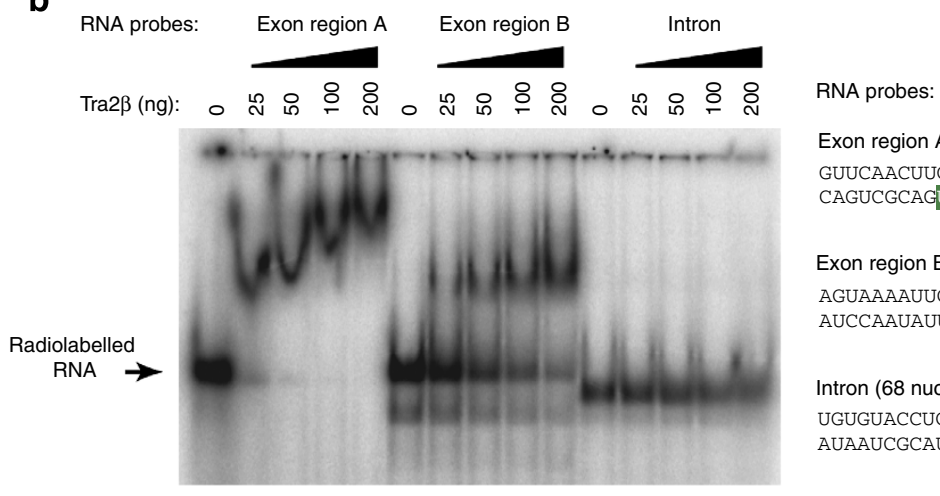

Exon region $\mathrm{A}$ (69 nucleotides)

GUUCAACUUGCUGUGAAUAGAGUAACUC

CAGUCGCAGUGAAGAUUGUAGAUAUGAAG

Exon region $\mathrm{B}$ ( 68 nucleotides)

AGUAAAAUUCUAUGGUCACAGGAGAGAAGGCAAU

AUCCAAUAUUUAUUUCUGGAGUACUGUAGUGGAG

Intron (68 nucleotides)

UGUGUACCUCUCCUUCACUACCUUUAGGGUGGCA AUAAUCGCAUCUACCCCAUAGGAGCUUAGGACAG

C

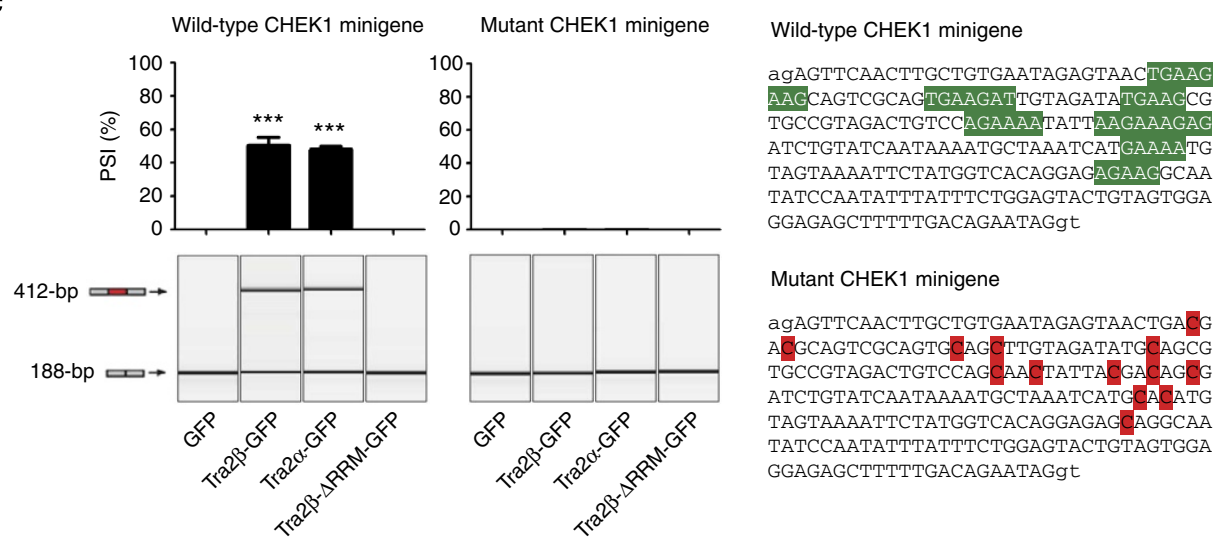

Figure 7 | Human Tra2 $\alpha$ and Tra2 $\beta$ regulate CHEK1 exon 3 through direct RNA protein interactions. (a) High resolution map CHEK1 exon 3 showing mapped iCLIP tags (purple bars) and the three subregions of the pre-mRNA, which were used to generate RNA probes for EMSAs. This screenshot was downloaded from the UCSC genome browser ${ }^{35}$. (b) Molecular interactions between purified Tra2 $\beta$ protein and RNA probes in and around CHEK1 exon 3 (the location of these probes is shown in part $\mathbf{a}$ ). The sequences of the probes are shown to the right, with predicted Tra2 $\beta$ binding sites shaded green. (c) Splicing patterns of mRNAs made from a minigene containing CHEK1 exon 3 in response to coexpressed fusion proteins, expressed either as a PSI (upper bar chart, $n=3$ independent experiments) or shown as one of the original capillary electrophoresis gel-like images from a single experiment (lower image). The sequence of CHEK1 exon 3 is shown to the right, with the predicted Tra2 $\beta$ binding sites shaded green (above) and the altered sequence after these sites were mutated (below, the altered nucleotides are shaded red). Probability $(P)$ values were calculated using an independent two-sample $t$-test between PSI levels of the minigene-derived CHEK1 exon 3 in cells cotransfected with GFP and each of the different Tra2 constructs (statistical significance: ${ }^{\star} P<0.05,{ }^{\star \star} P<0.01,{ }^{\star \star \star} P<0.0001$ ). All data represented by bar charts was generated from three biological replicates and error bars represent s.e.m.

abolished splicing activation in response to coexpressed Tra2 proteins.

Tra2 proteins are required for $\mathrm{CHK} 1$ protein expression. We carried out further experiments to test if Tra2 proteins are also required for expression of full-length $\mathrm{CHK} 1$ protein. On western blots, we could detect expression of a single major protein $\mathrm{CHK} 1$ isoform in MDA-MB-231 cells, corresponding to the expected size of full-length CHK1 protein $(54 \mathrm{kDa})$. This band was substantially reduced following treatment with an siRNA directed against CHEK1 mRNA (Fig. 8a). Consistent with joint control of CHEK1 expression by Tra2 $\alpha$ and Tra2 $\beta$, levels of full-length CHK1 protein were also substantially reduced after joint depletion of Tra $2 \alpha$ and Tra2 $\beta$. Expression of full-length CHK1 protein was also reduced after joint $\operatorname{Tra} 2 \alpha$ and $\operatorname{Tra} 2 \beta$ protein depletion in MCF7, PC3 and to a lesser extent HeLa cells (Supplementary Fig. 7a).

A shorter isoform of the CHK1 protein (termed CHK1-S) has previously been reported to be translated from an alternative downstream translational initiation site in exon 3-skipped CHEK1 $\mathrm{mRNA}^{37}$. In our experiments, although depletion of Tra2 proteins switched splicing of CHEK1 exon 3, they did not lead to an observable increase in any shorter isoform of the CHK1 protein. We detected much lower expression levels of possible shorter CHK1 protein isoforms on western blots compared with full-length $\mathrm{CHK} 1$ protein (the $\sim 43 \mathrm{KDa}$ protein that would correspond in size to CHK1-S could only be seen on long 
a

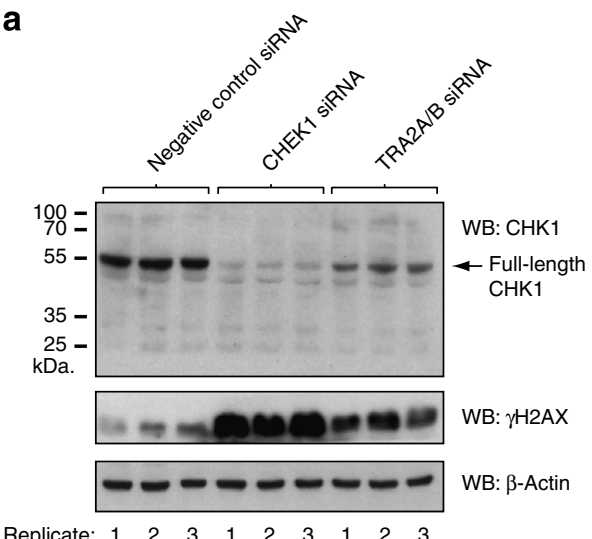

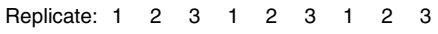

d

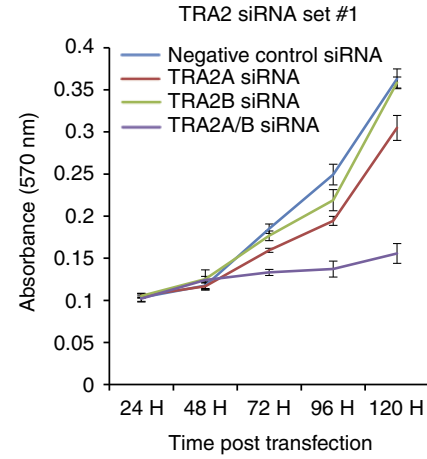

b

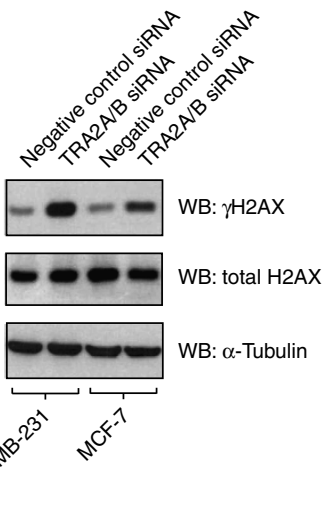

C

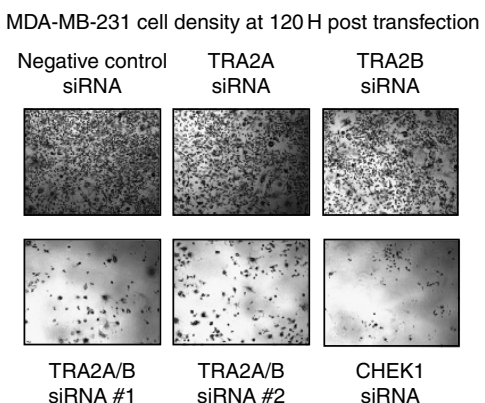

e

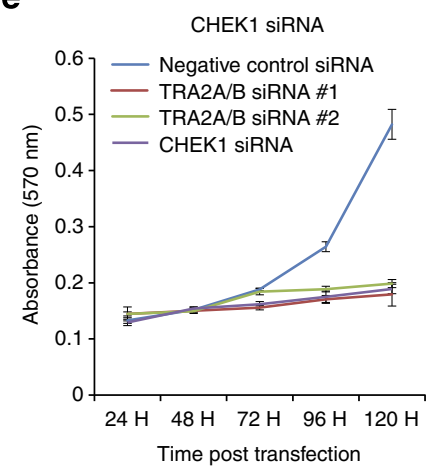

g
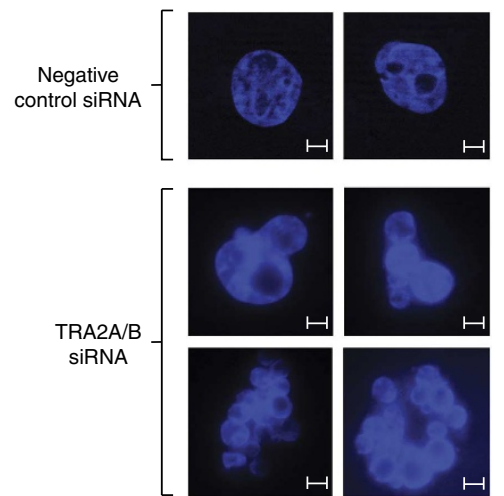

f
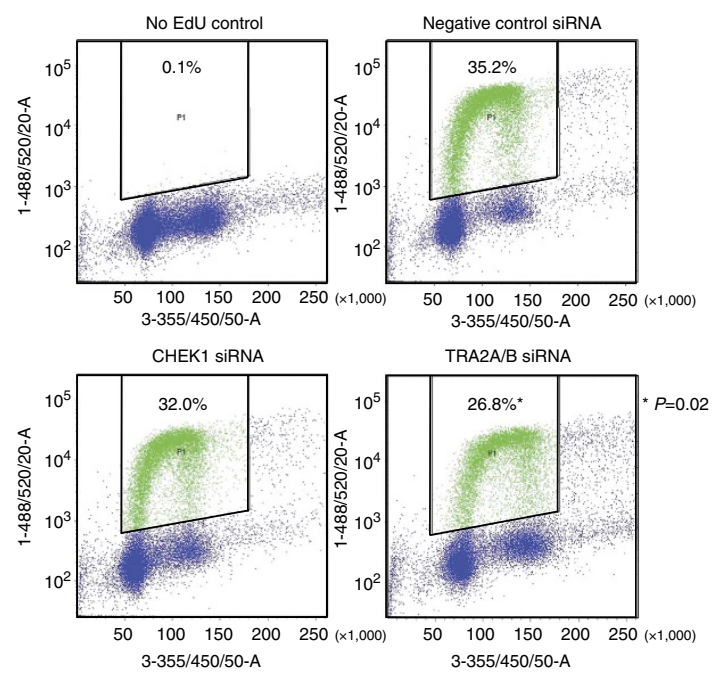

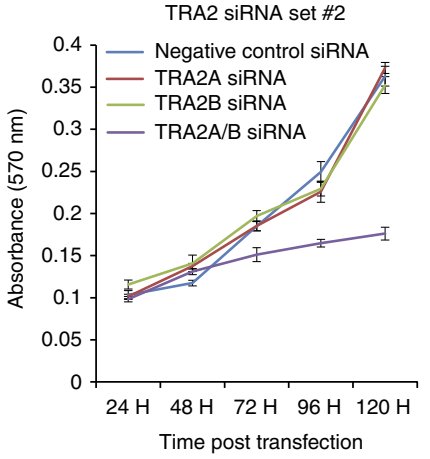

Negative control siRNA

Figure 8 | Human Tra2 proteins are essential for expression of full-length CHK1 protein and to maintain cell viability. (a) Full-length CHK1 protein expression is depleted by siRNAs specific to CHEK1 mRNA and also by joint siRNAs specific to the TRA2A and TRA2B mRNAs. In each case, samples from three independent replicate experiments were analysed in parallel. Also detected in these samples are levels of $\gamma \mathrm{H} 2 \mathrm{AX}$ and $\alpha$-tubulin. (b) Expression of total $\mathrm{H} 2 \mathrm{AX}$ and $\gamma \mathrm{H} 2 \mathrm{AX}$ after joint Tra2 $\alpha$ and Tra2 $\beta$ depletion, or depletion with a control siRNA in MDA-MB-231 cells and MCF7 cells. (c) Measurement of cell density $120 \mathrm{~h}$ after transfection of siRNAs targeting different regions of the TRA2A and TRA2B mRNAs or CHEK1 mRNA. (d) Joint (but not single) depletion of Tra2 $\alpha$ and Tra2 $\beta$ proteins reduced MDA-MB-231 cell viability measured by MTT assays after siRNA transfection. (e) Depletion of CHK1 protein alone was sufficient to reduce viability of MDA-MB-231 cells measured by MTT assay at different time points after siRNA transfection. (f) Joint depletion of Tra2 $\alpha$ and Tra2 $\beta$ reduced the proportion of EdU-positive MDA-MB-231 cells $96 \mathrm{~h}$ after siRNA transfection. Separate panels, shown clockwise from top left, show fluorescence-activated cell sorting analysis of control MDA-MB-231 cells incubated without EdU; cells transfected with a negative control siRNA and incubated with EdU; cells transfected with siRNAs specific for TRA2A and TRA2B and incubated with EdU; and cells transfected with a single siRNA specific to CHEK1 and incubated with EdU. Probability $(P)$ values were calculated using an independent two-sample $t$-test comparing the percentage of EdU-positive cells of negative control siRNAtreated cells and the gene-specific siRNA-treated cells (statistical significance: ${ }^{\star} P<0.05,{ }^{\star \star} P<0.01,{ }^{\star \star \star} P<0.0001$ ). Data were generated from three biological replicates. (g) Examples of abnormal nuclear morphology observed within cells transfected with siRNAs specific for TRA2A and TRA2B (lower panel) compared with the normal morphology seen in negative control siRNA-treated cells (upper panel). Cells were stained with 4',6-diamidino-2-phenylindole, and these images were taken $96 \mathrm{~h}$ after siRNA transfection. The scale bar shows $5 \mu \mathrm{M}$. Uncropped western blots are shown in Supplementary Figs $11-15$. 
exposure, and decreased on siRNA treatment,Supplementary Fig. 7b). CHK1-S protein is reported to be regulated over the cell cycle and in tumours ${ }^{37}$. To test for cell cycle regulated splicing inclusion of CHEK1 exon 3, we prepared RNAs from KG1 cell populations enriched in different cell cycle stages prepared using elutriation (Supplementary Fig. 5). When analysed by RT-PCR, very similar patterns of CHEK1 exon 3 splicing inclusion were observed in each of the cell populations even though they contain different cell cycle profiles. We could detect high levels of both CHEK1 splice isoforms in RNA purified from a small panel of breast cancer tissues, although we did not see an enrichment of either isoform in any particular tumour type at the RNA level (Supplementary Fig. 6). Overall, the above data are most consistent with Tra2 $\alpha$ and $\operatorname{Tra} 2 \beta$ activity being essential for expression of full-length CHK1 protein rather than inducing expression of a shorter protein isoform of CHK1.

Tra2 protein depletion affects DNA damage and cell viability. Although it is not as a direct target of CHK1 phosphorylation, $\gamma \mathrm{H} 2 \mathrm{AX}$ has been used as a marker for the replication stress that can be induced by depleted CHK1 levels ${ }^{38,39}$. Similar to previous observations $^{38}$, we observed greatly increased levels of the DNA damage marker $\gamma \mathrm{H} 2 \mathrm{AX}$ following depletion of CHK1 protein by siRNA, compared with cells treated with a negative control siRNA (Fig. 8a). Increased $\gamma \mathrm{H} 2 \mathrm{AX}$ levels were also observed after joint depletion of $\operatorname{Tra} 2 \alpha$ and Tra2 $\beta$ proteins in MDA-MB-231 cells and in MCF7 cells (Fig. 8a,b). The relative increased levels of $\gamma \mathrm{H} 2 \mathrm{AX}$ following CHEK1 or TRA2A/B siRNA treatment appear proportional to the reduction in full-length $\mathrm{CHK} 1$ protein expression observed by western blot.

Microscopy and MTT assays also indicated reduced cell viability 120 hours after joint $\operatorname{Tra} 2 \alpha$ and $\operatorname{Tra} 2 \beta$ depletion (Fig. 8c,d). In contrast, single depletion of either $\operatorname{Tra} 2 \alpha$ or $\operatorname{Tra} 2 \beta$ had negligible effect on cell viability compared with mock depleted cells. Similar results were obtained using two independent sets of siRNAs targeted at different regions of the mRNAs. This reduction in cell viability from joint removal of $\operatorname{Tra} 2 \alpha$ and $\operatorname{Tr} 2 \beta$, compared with the negligible effects of removing either protein alone, suggest that $\operatorname{Tra} 2 \alpha$ and $\operatorname{Tra} 2 \beta$ are functionally interchangeable for maintaining cell viability in MDA-MB-231 cells, as well as in splicing control.

Depletion of CHK1 also reduced cell viability in MDA-MB-231 cells (Fig. 8e). This suggests that depletion of full-length CHK1 protein would likely be sufficient by itself to contribute to the loss of cell viability observed after joint $\operatorname{Tra} 2 \alpha$ and $\operatorname{Tra} 2 \beta$ depletion. To test if re-introduction of full-length CHK1 protein would be sufficient to restore viability of joint $\operatorname{Tra} 2 \alpha$ and $\operatorname{Tra} 2 \beta$ proteindepleted cells, we made a stable cell line in the FLP-in HEK-293 cell background in which a full-length FLAG-tagged CHK1 protein was expressed under control of a tetracycline promoter. Similar to the result obtained in MDA-MB-231 cells, joint depletion of $\operatorname{Tr} 2 \alpha$ and $\operatorname{Tra} 2 \beta$ reduced cell viability in this stable HEK-293 cell line. However, although the full-length FLAGtagged CHK1 protein was efficiently induced by tetracycline, it was not sufficient to rescue cell viability after joint Tra2 protein depletion (Supplementary Figs 8 and 9). While we cannot rule out that the tagged full-length CHK1 protein failed to rescue viability of this cell line for another reason, this result is consistent with multiple exons controlled by Tra2 proteins (including CHEK1 exon 3) being important for cell viability.

Finally, we monitored incorporation of the thymidine analogue EdU using flow cytometry to determine whether joint Tra2 protein depletion affected cell proliferation of MDA-MB-231 cells (Fig. 8f). After joint Tra2 protein depletion, we observed a significant reduction in the proportion of EdU-positive cells $96 \mathrm{~h}$ after siRNA transfection (an $8.4 \%$ reduction, $P=0.02$ ), indicating fewer cells had initiated DNA replication after joint Tra2 protein depletion. A slight reduction in the proportion of EdU-positive cells was observed after single CHK1 protein depletion, but this was not statistically significant when compared with negative control siRNA-treated cells. Joint Tra2 protein depletion also caused an increase in the proportion of cells containing abnormally shaped nuclei $96 \mathrm{~h}$ after siRNA transfection, consistent with major biological defects (Fig. 8g).

\section{Discussion}

Here we find that only joint depletion of both $\operatorname{Tra} 2 \alpha$ and $\operatorname{Tr} 2 \beta$ proteins (and not single depletion of either protein alone) could induce substantial splicing switches in endogenous Tra2 $\beta$ target exons in MDA-MB-231 cells. This joint depletion strategy has enabled us to derive the most comprehensive map of dual Tra2dependent target exons in any organism to date. Among the jointly regulated exons identified here was a key exon in the CHEK1 gene, which encodes a protein essential for monitoring DNA damage and controlling cell cycle progression ${ }^{37,39}$. Exon 3 of the CHEK1 gene is 224 nucleotides long; hence, skipping of this exon in the absence of $\mathrm{Tra} 2$ proteins would frameshift the reading frame of the CHEK1 mRNA if a downstream translational initiation site is not selected ${ }^{37}$. Joint depletion of both Tra2 proteins quantitatively switched CHEK1 pre-mRNA splicing, reduced expression of full-length CHK1 protein, and led to an increase in DNA damage as monitored by accumulation of $\gamma \mathrm{H} 2 \mathrm{AX}$. We also confirmed $S M N 2$ exon 7 as a joint $\operatorname{Tra} 2 \alpha / \operatorname{Tra} 2 \beta$ target exon. Joint control by $\operatorname{Tra} 2 \alpha$ provides an explanation why SMN2 exon 7 is a target for Tra2 $\beta$ in transfected cells, but not appreciably affected in Tra2b single knockout mice ${ }^{15}$.

This strategy also reveals that Tra2 proteins are required for splicing inclusion of some constitutively spliced exons. To the best of our knowledge, Tra2 proteins have only previously been described as alternative splicing factors ${ }^{26}$. Low levels of apparent alternative splicing of constitutive exons might be ascribed to error prone exon recognition by the spliceosome ${ }^{40}$. However, the ANKRD1 and SMC4 exons are not annotated as alternatively spliced in any tissue consistent with them being true constitutive exons, yet also show high-amplitude splicing changes upon Tra2 protein depletion. A role in constitutive splicing brings the Tra2 proteins closer to the core SR group in described molecular functions $^{23}$. Consistent with this newly discovered role, Tra2 $\beta$ protein is fairly evenly expressed across mouse tissues, so would be available in most cells for splicing inclusion of constitutive exons ${ }^{10,18}$. Although we only detected exons activated by Tra2 proteins in this study, exons have previously been described that are repressed by Tra2 proteins ${ }^{29,4}$. Such repressed exons might have either eluded our search criteria or occur less frequently.

Genes containing Tra2-dependent exons are enriched in processes associated with chromosome biology. Although GO annotations are known to be incomplete and can differ in accuracy $^{42}$, this pattern of functional enrichment observed in our data is notably coherent. A potential association between the Tra2-dependent exons and chromosome biology is additionally supported by the connectivity of the BioGRID functional interaction network. In addition to the regulated CHEK1 alternative splice, $6 / 17$ of the strongest $\operatorname{Tr} 2$ protein-responsive exons (showing $>40$ point PSI change after Tra2 depletion) were also in genes involved in chromosome structure and epigenetic regulation, including MSL3 ( -41 point PSI exon switch after Tra2 depletion). MSL3 is the human ortholog ${ }^{43}$ of the Drosophila melanogaster MSL3 gene, which regulates chromatin remodelling during sex determination, and is under control of Sex Lethal (a protein just upstream of Tra2 in the Drosophila sex determination 
pathway) in flies. The SMC4 gene ( -66 point PSI exon switch after Tra2 depletion) encodes a protein important for DNA repair and chromosome condensation, and also interacts with the CTCF transcription factor that modifies chromatin structure ${ }^{44}$. The ANKRD1 ( -73 point PSI exon switch after Tra2 depletion) encodes a transcription factor, which is a negative regulator of cardiac genes ${ }^{45}$. Also among the genes with highly Tra2 proteinresponsive exons was GLYR1 ( -41 point PSI exon switch after Tra2 depletion), which is a cofactor for histone demethylation ${ }^{46}$, and the zinc-finger protein ZCCHC9 ( -68 point PSI exon switch after Tra2 depletion), which targets histone mRNAs for degradation ${ }^{47}$. ZCCHC7 ( -72 point PSI exon switch after Tra2 depletion) and ZCCHC11 ( -46 point PSI exon switch after Tra2 depletion) encode zinc-finger proteins homologous to ZCCHC9, but with roles in non-coding RNA metabolism ${ }^{47-49}$. The MPHOSPH10 gene ( -42 point PSI exon switch after Tra2 depletion) encodes a protein involved in ribosomal RNA processing in interphase, and is associated with chromosomes during mitosis ${ }^{50}$. Other genes involved in chromatin modification that are controlled by Tra2 proteins but did not fit into the most responsive group include the NASP (NASP-T, -12 point PSI exon switch after Tra2 depletion) and ATRX genes ( -23 point PSI exon switch after Tra2 depletion) ${ }^{18,51}$. Interestingly, the two known Drosophila Tra2 splicing targets Doublesex and Fruitless are both transcription factors ${ }^{12}$, and one of the major functions of the Drosophila sex determination pathway is dosage compensation via chromatin modification. Our data lend further support to the association of particular splicing regulators with the regulation of coherent cellular functions, also described for NOVA, RBFox2, PTB and T-STAR ${ }^{4-7}$.

Our data indicate a high degree of functional redundancy between $\operatorname{Tra} 2 \alpha$ and $\operatorname{Tra} 2 \beta$, and a powerful homeostatic repressive feedback activity of Tra $2 \beta$ over Tra $2 \alpha$ that buffers splicing changes when just one of these proteins is missing. Although splicing defects after single Tra2 $\alpha$ and Tra2 $\beta$ depletion were small, they were often individually statistically significant (for example, in the ATRX gene). Such fine tuning of splicing profiles by joint Tra 2 protein concentration by splicing feedback control might be important in whole organisms and at particular points of development (for example, in brain or testis development). Even individually, small splicing defects over many Tra2 $\beta$-target exons might cumulatively cause physiological defects. This might explain why Tra2b-null mice are embryonic lethal despite containing a Tra2a gene $\mathrm{e}^{15,17,18}$. Similar asymmetric expression patterns, in which a dominantly expressed splicing factor crossregulates other family member proteins, have been found in the PTB family, where PTBP1 cross-regulates PTBP2 and PTBP3 (ref. 53). Comprehensive identification of PTBP1 targets similarly required joint depletion of PTBP1 and PTBP2 (ref. 53) Future studies of Tra $2 \beta$-regulated splicing may also benefit by considering expression levels of both Tra2 proteins, rather than in the context of Tra2 $\beta$ expression alone.

Joint depletion of both Tra2 protein levels reduced cell viability in MDA-MB-231 cells, likely at least in part because of the requirement for productive splicing of the CHEK1 mRNA. CHK1 protein expression is critical to reduce replication stress in cancer cells undergoing rapid proliferation driven by oncogenes including RAS and $M Y C^{54,55}$. Our data thus suggest the Tra2 proteins may represent novel targets to inhibit cancer cell growth.

\section{Methods}

Cell culture. MDA-MB-231 cells and MCF7 cells were maintained in DMEM (no phenol red) plus $10 \%$ fetal bovine serum and $1 \%$ Penicillin Streptomycin. HEK-293, HeLa and PC3 cells were maintained in DMEM plus 10\% fetal bovine serum. Cells lines were originally purchased from the American Type Culture Collection and LGC Standards, Europe.
iCLIP. Triplicate iCLIP experiments were performed following the iCLIP proto$\mathrm{col}^{33}$. Briefly, MDA-MB-231 cells were irradiated with $400 \mathrm{~mJ} \mathrm{~cm}{ }^{-2}$ ultraviolet-C light on ice, lysed and subject to partial RNase digestion. The crosslinked Tra2 $\beta$-RNA complexes were then immunoprecipitated using Protein A Dynabeads (Invitrogen) and a rabbit polyclonal anti-Tra2 $\beta$ antibody (Abcam, ab31353). cDNA libraries were prepared according to the published iCLIP protocol. High throughput sequencing of cDNA libraries was performed using an Illumina GAIIx.

RNA-seq. RNA was extracted from cells using RNeasy Plus Mini Kit (Qiagen) following manufacturer's instructions and re-suspended in nuclease-free water. All RNA samples were DNase treated using DNA-free kit (Invitrogen) and stored at $-80^{\circ} \mathrm{C}$ prior to RNA quality control check using 2100 Agilent Bioanalyser and mRNA library prep using TruSeq mRNA library kit (Illumina). Pair-end sequencing was done in total for six samples (three biological replicates of negative control siRNA-treated cells and three biological replicates from TRA2A and TRA2B siRNA-treated cells) using an Illumina HiSeq 2000.

Bioinformatics (iCLIP and RNA-seq analysis). iCLIP data analysis, crosslink site identification and quantification, randomization of iCLIP positions and pentamer enrichment analysis were performed according to published procedures ${ }^{33}$. Briefly, we used the human genome annotation version hg19, and gene annotations from Ensembl 59. Experiment barcode and random barcodes were registered and removed from iCLIP reads. After trimming, we ignored reads shorter than 11 nucleotides. Remaining trimmed reads were then mapped using Bowtie ${ }^{56}$, allowing two mismatches and accepting only reads with single hits. Crosslink sites were initially identified as the first nucleotide upstream of the iCLIP tag, and then filtered to determine statistically significant crosslink sites and those which occurred in clusters within 15 nucleotides windows and with a significant iCLIP tag count, compared with randomized positions, as described in Konig et al. ${ }^{56}$. For RNA-seq analysis, the base quality of raw sequencing reads were checked with FastQC (ref. 57) and refined with Seqtk (ref. 58) and Trim-galore (ref. 59). Reads were mapped to the hg19 reference with Tophat2 (ref. 60) and matches analyzed with Bedtools (ref. 61). Differentially expressed genes and exon usage were determined with DESeq (ref. 62) and DEXSeq (ref. 63) respecitvely.

siRNA transfection. Efficient knockdown of endogenous Tra $2 \alpha$, Tra $2 \beta$ and $\mathrm{CHK} 1$ proteins were achieved by transfecting cells with Silencer Select Pre-designed siRNAs (Ambion), targeting TRA2A mRNA (Ambion IDs: s26664 and s26665), TRA2B mRNA (Ambion IDs: s12749 and s12751) or CHEK1 mRNA (Ambion ID: s503) respectively, with siPORT NeoFX Transfection Agent (Ambion). Control cells were transfected with a negative control siRNA (Ambion Cat\#: 4390843). MDA-MB-231 cells grown in $100 \mathrm{~mm}$ tissue culture dishes were transfected with either $24 \mu \mathrm{l}$ of $10 \mu \mathrm{M}$ negative control siRNA (control), $12 \mu \mathrm{l}$ of $10 \mu \mathrm{M}$ siRNA targeting TRA2A, TRA2B or CHEK1 (single Tra $2 \alpha$, Tra2 $\beta$ or CHK1 knockdown) or $12 \mu \mathrm{l}$ of $10 \mu \mathrm{M}$ siRNA targeting TRA2A and $12 \mu \mathrm{l}$ of $10 \mu \mathrm{M}$ siRNA targeting TRA $2 B$ (joint Tra $2 \alpha$ and Tra2 $\beta$ knockdown). Cells were incubated for $72 \mathrm{~h}$ post siRNA transfection, before RNA extraction or western blotting.

Splicing assays: RNA extraction, RT-PCR and PCR. RNA was extracted using standard Trizol RNA extraction. cDNA was synthesized from $500 \mathrm{ng}$ total RNA in a $10 \mu \mathrm{l}$ reaction, using Superscript VILO cDNA synthesis kit (Invitrogen) following manufacturer's instructions. Splicing profiles were monitored by PCR using primers in flanking exons. For each PCR, $1 \mu \mathrm{l}$ diluted cDNA $(1 / 8)$ was used as template in a $10 \mu \mathrm{l}$ PCR reaction using Phusion High-Fidelity PCR Kit (NEB, UK) following manufacturer's instructions. Splicing profiles were monitored and quantified using the Qiaxcel capillary electrophoresis system (Qiagen) and PSI was calculated as described previously ${ }^{18}$. All primers used for splicing assays are provided in Supplementary Data 3.

Quantitative PCR. Relative gene expression was determined by quantitative real time PCR using the SYBR Green PCR Master Mix kit (Applied Biosystems) and an Applied Biosystems 7900HT Fast Real-Time PCR Machine. cDNA was generated from equal quantities of total RNA for each sample using Superscript VILO cDNA synthesis kit (Invitrogen) following manufacturer's instructions. Gene expression was calculated relative to three housekeeping genes ACTB, GAPDH and TUBB. $C_{t}$ values for each sample were calculated using SDS 2.4 software (Applied Biosystems) and relative mRNA expression was calculated using the $2^{-\Delta \Delta C t}$ method.

Calculation of Tra2 $\beta$ binding site density. Tra2 $\beta$ binding site density was calculated as the percentage of nucleotides within an exon that correspond to the top 10 kmers identified from the Tra2 $\beta$ iCLIP experiments (Supplementary Fig. 2b).

Detection of proteins using western blotting. Endogenous proteins were detected by western blot analysis using the following primary antibodies and dilutions: Tra2 $\alpha$ (Novus Biologicals, H00029896-B01P;1:500 dilution), Tra2 $\beta$ (Abcam, ab31353;1:2,000 dilution), CHEK1 (Proteintech, 10362-1-AP;1:250 dilution), Histone H2AX (Santa Cruz Biotechnology, sc54-606;1:500 dilution), 
$\gamma \mathrm{H} 2 \mathrm{AX}$ (Ser 139) (Santa Cruz Biotechnology, sc-101696;1:500 dilution), FLAG (Sigma-Aldrich, F3040;1:2,000 dilution), $\beta$-Actin (Sigma-Aldrich, A5441,1:2,000 dilution) and $\alpha$-Tubulin (Sigma-Aldrich, T5168;1:2,000 dilution).

ANKRD1 and CHEK1 minigene construction and mutagenesis. The ANKRD1
constitutive exon and $\sim 200$ nucleotides of flanking intronic region was amplifie from human genomic DNA using the cloning primers ANKRD1 F (5'-AAAA AAAAAGAATTCAAAATCTAAGACTTGCTTATGGCATT- $3^{\prime}$ ) and ANKRD1 R (5'AAAAAAAAAGAATTCAGCATGAGAGTTACCGTGAGC- $3^{\prime}$ ). The PCR products were digested with $\mathrm{BamH1}$ restriction enzyme and cloned into the $\mathrm{pXJ} 41$ vector $^{64}$ using the $M f e 1$ site midway through the 757 nucleotide rabbit $\beta$-globin intron 2 . Tra2 $\beta$ binding site mutations were made using site directed mutagenesis with the following primers; ANKRD1 M1F (5'- AGAACACATATCAAAGCTT GCACATTTATACGACCTTGAAA- ${ }^{\prime}$ ), ANKRD1 M1R (5'-CAAGGTCGTATAA ATGTGCAAGCTTTGATATGTGTTCTAG- $3^{\prime}$ ), ANKRD1 M2F (5'-ATCATT CAACTGCAGCAACGGCAACAATACAGGCACACTAAAG-3') and ANKRD1 M2R (5'-GAACTTTAGTGTGCCTGTATTGTTGCCGTTGCTGCAGTTGAA TG- $\left.3^{\prime}\right)$. The CHEK1 alternative exon and approximately 250 nucleotides of flanking intronic region was synthesised in vitro and similarly cloned into the pXJ41 vector. A mutated version that disrupted Tra2 $\beta$ binding sites was also synthesised (sequence provided in Fig. 7c) and cloned into the pXJ41 vector. Analysis of splicing patterns of mRNAs transcribed from minigenes was carried out in HEK-293 cells as previously described ${ }^{18,24}$, using primers within the $\beta$-globin exons of pXJ41; PXJRTF ( $5^{\prime}$-GCTCCGGATCGATCCTGAGAACT-3') and PXJB (5'-GCTGCAATAAACAAGTTCTGCT-3').

EMSAs. Gel shift experiments ${ }^{18,65}$ were performed using full-length Tra2 $\beta$ protein and in vitro-translated RNA probes made from constructs containing amplified regions of the human CHEK1 gene, cloned into the pBluescript vector. Three regions of the human CHEK1 gene were amplified using the following primers: CHEK1 intronic $\mathrm{F}$ ( $5^{\prime}$-AAAAAAAAAGGTACCTGTGTACCTCTCCTTCACTA CC- $\left.3^{\prime}\right)$, CHEK1 intronic R ( $5^{\prime}$-AAAAAAAAAGAATTCCTGTCCTAAGCTCCT ATGGGG-3'), CHEK1 exon region A F ( $5^{\prime}$-AAAAAAAAAGGTACCgttcaacttgc tgtgaatagagt- $\left.3^{\prime}\right)$, CHEK1 exon region A R ( $5^{\prime}$-AAAAAAAAAGAATTCggcacg CTTCAtatctacaATCT-3'), CHEK1 exon region B F (5'-AAAAAAAAAGGTACC agtaaaattctatggtcacagga- $\left.3^{\prime}\right)$ and CHEK1 exon region B R ( $5^{\prime}$-AAAAAAAAAGAA TTCctccactacagtactccagaaat- $\left.3^{\prime}\right)$.

GO and functional network analysis. $\mathrm{GO}^{66}$ enrichment analysis was carried out using the Bioconductor GOstats package version 2.24.0refs 67,68 . Enrichments of GO biological process terms were calculated using the conditional hypergeometric test with a significance cut-off of 0.001 and using a background of genes that are normally expressed in MDA-MB-231 cells. Annotations were taken from the Bioconductor Homo sapiens annotation package org.Hs.eg.db version 2.8.0ref. ${ }^{69}$ The analysis was run in the open source statistical package $\mathrm{R}$ version 3.0 .1 ref. ${ }^{70}$.

Interaction data for Homo sapiens was retrieved from the BioGRID database (version 110). These data were integrated into a network in which nodes represented genes or gene products, and edges represented any type of BioGRID interaction between the nodes. The network was visualised using the Cytoscape visualization platform ${ }^{71}$, and was coloured based on annotations to top five enriched GO biological processes (as downloaded from QuickGO ${ }^{71}$ ). Where a protein was annotated to more than one term, the most specific annotation was chosen.

MTT assay. MTT assays were performed using MTT Cell Proliferation Assay Kit (Cayman Chemical), following manufacturer's instructions. An siRNA transfection mix was added to a suspension of $\sim 2 \times 10^{5}$ MDA-MB- 231 cells in $10 \mathrm{ml}$ media. The siRNA/cell suspension was gently mixed and a $100 \mu \mathrm{l}$ aliquot was added per well to a 96-well plate. Absorbance from the MTT assay was measured at 24, 48, 72, 96 and $120 \mathrm{~h}$ after siRNA transfection/seeding of cells. Relative density of cells was also compared $120 \mathrm{~h}$ after seeding cells by microscopy.

Fluorescence-activated cell sorting analysis of EdU-positive cells. MDA-MB231 cells were incubated with $10 \mu \mathrm{M}$ EdU for $4 \mathrm{~h}, 96 \mathrm{~h}$ after siRNA transfection. Cell fixation, permeabilization and EdU detection was performed using the Click-iT EdU Flow Cytometry Assay Kit (Life Technologies) following the manufacturer's instructions. Data were collected and analysed using a BD LSR II flow cytometer using $488 \mathrm{~nm}$ excitation and a 520/20 band-pass for detection of EdU Alexa Fluor488 azide and $355 \mathrm{~nm}$ excitation and a 450/50 band-pass for detection of 4',6-diamidino-2-phenylindole. Experiments were performed with biological triplicate samples and 30,000 cells were analysed per sample. A no-EdU control sample was used to inform our gating strategy to calculate the proportion of EdUpositive cells.

Analysis of nuclear morphology. To investigate nuclear morphology, cells were fixed with $4 \%$ paraformaldehyde followed by nuclear staining with 4,6-diamidino2-phenylindole, $96 \mathrm{~h}$ after siRNA transfection (siRNA transfection as described above).
Elutriation and cell cycle evaluation. Elutriation: Cells were size fractionated by centrifugal elutriation, using flow rates of $10,13,17,20,24$ and $28 \mathrm{ml} \mathrm{min}^{-1}$ (ref. 72). Cell cycle evaluation: Cell cycle phase enrichment of cells was assessed using immunofluorescence staining for CENPF (late $S, G_{2}, G_{2} / M$ ) ${ }^{73}$ and phosphohistone $\mathrm{H} 3 \mathrm{~S} 10\left(\mathrm{G}_{2} / \mathrm{M}, \mathrm{M}\right)^{74}$. Asynchronous and elutriated $\mathrm{KGl}$ cells were suspended in PBS, spotted onto poly-lysine-coated slides and processed for immunofluorescence. Images were captured and cells were scored for CENPF and phospho-H3S10 staining.

Generation of tetracycline-inducible HEK-293 cells. Full-length CHK1-FLAG cDNA was amplified from the pcDNA4-Chk1-Flag plasmid (Addgene plasmid \#22894) using the primers CHEK1 FLAG F (5'-AAAAAAAAAGCGGCCGC atggcagtgccetttgtggaagac- $\left.3^{\prime}\right)$ and CHEK1 FLAG R (5'-AAAAAAAAAGTCGAC tcatgtggcaggaagccaaatcttc- $3^{\prime}$ ) and cloned into the Flp-In expression vector (pCDNA5). To generate an inducible cell line, the CHK1-FLAG-pCDNA5 vector was cotransfected with the Flp recombinase plasmid (pOG44) into Flp-In HEK-293 cells and selected for using treatment with Hygromycin B. Following Hygromycin B selection, CHK1-FLAG expression was induced by the addition of tetracycline to promote expression CHK1-FLAG expression via a tetracycline-inducible promoter.

\section{References}

1. Fox-Walsh, K. L. \& Hertel, K. J. Splice-site pairing is an intrinsically high fidelity process. Proc. Natl Acad. Sci. USA 106, 1766-1771 (2009).

2. Kelemen, O. et al. Function of alternative splicing. Gene 514, 1-30 (2013).

3. Djebali, S. et al. Landscape of transcription in human cells. Nature 489, 101-108 (2012).

4. Ehrmann, I. et al. The tissue-specific RNA binding protein T-STAR controls regional splicing patterns of neurexin pre-mRNAs in the brain. PLoS Genet 9, e1003474 (2013).

5. Venables, J. P. et al. RBFOX2 is an important regulator of mesenchymal tissue-specific splicing in both normal and cancer tissues. Mol. Cell Biol. 33, 396-405 (2013).

6. Ule, J. et al. Nova regulates brain-specific splicing to shape the synapse. Nat. Genet. 37, 844-852 (2005).

7. Zhang, C. et al. Defining the regulatory network of the tissue-specific splicing factors Fox-1 and Fox-2. Genes Dev. 22, 2550-2563 (2008).

8. Llorian, M. et al. Position-dependent alternative splicing activity revealed by global profiling of alternative splicing events regulated by PTB. Nat. Struct. Mol. Biol. 17, 1114-1123 (2010).

9. Tsuda, K. et al. Structural basis for the dual RNA-recognition modes of human Tra2-beta RRM. Nucleic Acids Res. 39, 1538-1553 (2011).

10. Elliott, D.J., Best, A., Dalgliesh, C., Ehrmann, I. \& Grellscheid, S. How does Tra2beta protein regulate tissue-specific RNA splicing? Biochem Soc Trans $\mathbf{4 0}$ 784-8 (2012)

11. Baker, B. S. Sex in flies: the splice of life. Nature 340, 521-524 (1989).

12. Forch, P. \& Valcarcel, J. Splicing regulation in Drosophila sex determination. Prog. Mol. Subcell. Biol. 31, 127-151 (2003).

13. Dauwalder, B., Amaya-Manzanares, F. \& Mattox, W. A human homologue of the Drosophila sex determination factor transformer-2 has conserved splicing regulatory functions. Proc. Natl Acad. Sci. USA 93, 9004-9009 (1996).

14. Beil, B., Screaton, G. \& Stamm, S. Molecular cloning of htra2-beta-1 and htra2-beta-2, two human homologs of tra-2 generated by alternative splicing. DNA Cell Biol. 16, 679-690 (1997).

15. Mende, Y. et al. Deficiency of the splicing factor Sfrs 10 results in early embryonic lethality in mice and has no impact on full-length SMN/Smn splicing. Hum. Mol. Genet. 19, 2154-2167 (2010).

16. Roberts, J. M. et al. Splicing factor TRA2B is required for neural progenitor survival. J. Comp. Neurol. 522, 372-392 (2014).

17. Storbeck, M. et al. Neuronal-specific deficiency of the splicing factor tra2b causes apoptosis in neurogenic areas of the developing mouse brain. PLOS ONE 9, e89020 (2014).

18. Grellscheid, S. et al. Identification of evolutionarily conserved exons as regulated targets for the splicing activator tra2beta in development. PLoS Genet. 7, e1002390 (2011).

19. Best, A. et al. Expression of Tra2 beta in cancer cells as a potential contributory factor to neoplasia and metastasis. Int. J. Cell Biol. 2013, 843781 (2013).

20. Hofmann, Y., Lorson, C. L., Stamm, S., Androphy, E. J. \& Wirth, B. Htra2-beta 1 stimulates an exonic splicing enhancer and can restore full-length SMN expression to survival motor neuron 2 (SMN2). Proc. Natl Acad. Sci. USA 97, 9618-9623 (2000).

21. Glatz, D. C. et al. The alternative splicing of tau exon 10 and its regulatory proteins CLK2 and TRA2-BETA1 changes in sporadic Alzheimer's disease. J. Neurochem. 96, 635-644 (2006).

22. Long, J. C. \& Caceres, J. F. The SR protein family of splicing factors: master regulators of gene expression. Biochem J 417, 15-27 (2009).

23. Zhou, Z. \& Fu, X. D. Regulation of splicing by SR proteins and SR protein-specific kinases. Chromosoma 122, 191-207 (2013). 
24. Venables, J. P. et al. Up-regulation of the ubiquitous alternative splicing factor Tra2beta causes inclusion of a germ cell-specific exon. Hum. Mol. Genet. 14, 2289-2303 (2005).

25. Busch, A. \& Hertel, K. J. Evolution of SR protein and hnRNP splicing regulatory factors. Wiley Interdiscip. Rev. RNA 3, 1-12 (2012).

26. Tacke, R., Tohyama, M., Ogawa, S. \& Manley, J. L. Human Tra2 proteins are sequence-specific activators of pre-mRNA splicing. Cell 93, 139-148 (1998).

27. Clery, A. et al. Molecular basis of purine-rich RNA recognition by the human SR-like protein Tra2-beta1. Nat. Struct. Mol. Biol. 18, 443-450 (2011).

28. Uren, P. J. et al. Site identification in high-throughput RNA-protein interaction data. Bioinformatics 28, 3013-3020 (2012).

29. Anderson, E. S. et al. The cardiotonic steroid digitoxin regulates alternative splicing through depletion of the splicing factors SRSF3 and TRA2B. RNA 18, 1041-1049 (2012).

30. Lareau, L. F., Inada, M., Green, R. E., Wengrod, J. C. \& Brenner, S. E. Unproductive splicing of SR genes associated with highly conserved and ultraconserved DNA elements. Nature 446, 926-929 (2007).

31. Stoilov, P., Daoud, R., Nayler, O. \& Stamm, S. Human tra2-beta1 autoregulates its protein concentration by influencing alternative splicing of its pre-mRNA. Hum. Mol. Genet. 13, 509-524 (2004).

32. Saltzman, A.L., Pan, Q. \& Blencowe, B. J. Regulation of alternative splicing by the core spliceosomal machinery. Genes Dev 25, 373-84 (2011).

33. Konig, J. et al. iCLIP--transcriptome-wide mapping of protein-RNA interactions with individual nucleotide resolution. J. Vis. Exp. 50: pii 2638 (2011).

34. Dreszer, T. R. et al. The UCSC Genome Browser database: extensions and updates 2011. Nucleic Acids Res. 40, D918-D923 (2012).

35. Meyer, L. R. et al. The UCSC Genome Browser database: extensions and updates 2013. Nucleic Acids Res. 41, D64-D69 (2013).

36. Stark, C. et al. BioGRID: a general repository for interaction datasets. Nucleic Acids Res. 34, D535-D539 (2006).

37. Pabla, N., Bhatt, K. \& Dong, Z. Checkpoint kinase 1 (Chk1)-short is a splice variant and endogenous inhibitor of Chk1 that regulates cell cycle and DNA damage checkpoints. Proc. Natl Acad. Sci. USA 109, 197-202 (2012).

38. Gagou, M. E., Zuazua-Villar, P. \& Meuth, M. Enhanced H2AX phosphorylation, DNA replication fork arrest, and cell death in the absence of Chk1. Mol. Biol. Cell 21, 739-752 (2010).

39. Syljuasen, R. G. et al. Inhibition of human Chk1 causes increased initiation of DNA replication, phosphorylation of ATR targets, and DNA breakage. Mol. Cell Biol. 25, 3553-3562 (2005).

40. Melamud, E. \& Moult, J. Stochastic noise in splicing machinery. Nucleic Acids Res. 37, 4873-4886 (2009).

41. Chandler, D. S., Qi, J. L. \& Mattox, W. Direct repression of splicing by transformer-2. Mol. Cell Biol. 23, 5174-5185 (2003).

42. Skunca, N., Altenhoff, A. \& Dessimoz, C. Quality of computationally inferred gene ontology annotations. PLoS Comput. Biol. 8, e1002533 (2012).

43. Smith, E.R. et al. A human protein complex homologous to the Drosophila MSL complex is responsible for the majority of histone $\mathrm{H} 4$ acetylation at lysine 16. Mol Cell Biol 25, 9175-88 (2005).

44. Huang, K. et al. Ribosomal RNA gene transcription mediated by the master genome regulator protein CCCTC-binding factor (CTCF) is negatively regulated by the condensin complex. J Biol Chem 288, 26067-77 (2013)

45. Zolk, O. et al. Cardiac ankyrin repeat protein, a negative regulator of cardiac gene expression, is augmented in human heart failure. Biochem Biophys Res Commun 293, 1377-1382 (2002).

46. Fang, R. et al. LSD2/KDM1B and its cofactor NPAC/GLYR1 endow a structural and molecular model for regulation of H3K4 demethylation. Mol. Cell 49, 558-570 (2013).

47. Schmidt, M. J., West, S. \& Norbury, C. J. The human cytoplasmic RNA terminal U-transferase ZCCHC11 targets histone mRNAs for degradation. RNA 17, 39-44 (2011).

48. Fasken, M. B. et al. Air1 zinc knuckles 4 and 5 and a conserved IWRXY motif are critical for the function and integrity of the Trf4/5-Air1/2-Mtr4 polyadenylation (TRAMP) RNA quality control complex. J. Biol. Chem. 286, 37429-37445 (2011)

49. Hagan, J. P., Piskounova, E. \& Gregory, R. I. Lin 28 recruits the TUTase Zcchc11 to inhibit let-7 maturation in mouse embryonic stem cells. Nat. Struct. Mol. Biol. 16, 1021-1025 (2009).

50. Westendorf, J.M. et al. M phase phosphoprotein 10 is a human U3 small nucleolar ribonucleoprotein component. Mol Biol Cell 9, 437-49 (1998).

51. Eustermann, S. et al. Combinatorial readout of histone $\mathrm{H} 3$ modifications specifies localization of ATRX to heterochromatin. Nat. Struct. Mol. Biol. 18, 777-782 (2011)

52. Ule, J. et al. An RNA map predicting Nova-dependent splicing regulation. Nature 444, 580-586 (2006)

53. Spellman, R., Llorian, M. \& Smith, C. W. Crossregulation and functional redundancy between the splicing regulator PTB and its paralogs $\mathrm{nPTB}$ and ROD1. Mol. Cell 27, 420-434 (2007).
54. Bartek, J., Mistrik, M. \& Bartkova, J. Thresholds of replication stress signaling in cancer development and treatment. Nat. Struct. Mol. Biol. 19, 5-7 (2012).

55. Murga, M. et al. Exploiting oncogene-induced replicative stress for the selective killing of Myc-driven tumors. Nat. Struct. Mol. Biol. 18, 1331-1335 (2011).

56. Konig, J. et al. iCLIP reveals the function of hnRNP particles in splicing at individual nucleotide resolution. Nat Struct Mol Biol 17, 909-15 (2010).

57. Andrews, S. FastQC A quality control tool for high throughput sequence data. Available at http://www.bioinformatics.babraham.ac.uk/projects/fastqc/.

58. Li, H. seqtk. Available at https://github.com/lh3/seqtk.

59. Krueger, F. Trim Galore! Available at http://www.bioinformatics.babraham. ac.uk/projects/trim_galore/.

60. Kim, D. et al. TopHat2: accurate alignment of transcriptomes in the presence of insertions, deletions and gene fusions. Genome Biology 14, R36 (2013).

61. Quinlan, A.R. \& Hall, I.M. BEDTools: a flexible suite of utilities for comparing genomic features. Bioinformatics 26, 841-842 (2010).

62. Anders, S. \& Huber, W. Differential expression analysis for sequence count data. Genome Biology 11, R106 (2010).

63. Anders, S. et al. Detecting differential usage of exons from RNA-seq data. Genome Research 22, 2008-2017 (2012).

64. Bourgeois, C. F., Popielarz, M., Hildwein, G. \& Stevenin, J. Identification of a bidirectional splicing enhancer: differential involvement of SR proteins in 5' or 3' splice site activation. Mol. Cell. Biol. 19, 7347-7356 (1999).

65. Grellscheid, S. N. et al. Molecular design of a splicing switch responsive to the RNA binding protein Tra2beta. Nucleic Acids Res. 39, 8092-8104 (2011).

66. Ashburner, M. et al. Gene ontology: tool for the unification of biology. The Gene Ontology Consortium. Nat. Genet. 25, 25-29 (2000).

67. Falcon, S. \& Gentleman, R. Using GOstats to test gene lists for GO term association. Bioinformatics 23, 257-258 (2007).

68. Gentleman, R. C. et al. Bioconductor: open software development for computational biology and bioinformatics. Genome Biol. 5, R80 (2004).

69. Carlson, M. org.Hs.eg.db: Genome wide annotation for Human. R package version 2.8.0. (2012).

70. Rcoreteam. R: A language and environment for statistical computing. $\mathrm{R}$ Foundation for Statistical Computing, Vienna, Austria.

71. Shannon, P. et al. Cytoscape: a software environment for integrated models of biomolecular interaction networks. Genome Res. 13, 2498-2504 (2003).

72. Ly, T. et al. A proteomic chronology of gene expression through the cell cycle in human myeloid leukemia cells. Elife 3, e01630 (2014)

73. Liao, H., Winkfein, R. J., Mack, G., Rattner, J. B. \& Yen, T. J. CENP-F is a protein of the nuclear matrix that assembles onto kinetochores at late G2 and is rapidly degraded after mitosis. J. Cell Biol. 130, 507-518 (1995).

74. Hendzel, M. J. et al. Mitosis-specific phosphorylation of histone H3 initiates primarily within pericentromeric heterochromatin during G2 and spreads in an ordered fashion coincident with mitotic chromosome condensation. Chromosoma 106, 348-360 (1997).

\section{Acknowledgements}

We thank Professor Jernej Ule and Dr Julian Konig for help with the iCLIP analysis, and Professor Ian Eperon, Dr Ingrid Ehrmann and Dr Jennifer Munkley for comments on the manuscript. Andrew Best was a Breast Cancer Campaign funded PhD student. This project was supported by the Breast Cancer Campaign (Grant 2009NovPhd21), the Wellcome Trust (Grant numbers WT080368MA and WT089225/Z/09/Z), the BBSRC (grant numbers BB/D013917/1 and BB/I006923/1), a Canadian Institute of Health Research grant to B.C. (MOP93917) and the Leukaemia and Lymphoma Research Specialist Programme Grant 12031 (to C.A.A. and I.G.C). B.K. was a BHF Professor of Cardiology.

\section{Author contributions}

A.B. performed iCLIP, RNA-seq, splicing and cellular analyses, and performed experiments; K.J. performed GO and BioGrid analyses; C.D. performed EMSAs; E.H. helped with iCLIP experiments; M.K.-K. made stable HEK-293 cell lines; T.C. performed iCLIP sequence analysis; Y.X. and M.S.K. performed RNA-seq bioinformatic analysis; M.D. made minigenes; R.H. and B.K. performed iCLIP sequencing; A.W. helped with analysis; J.P.V., R.K. and B.C. analysed the role of multiple RNA binding proteins for CHEK1, SMC4, GLYR1 and ANKRD1; I.G.C., K.C.L. and C.A.A. purified and characterized KG1 cell fractions; A.B., A.T.-C. and D.J.E. were involved in study design and data analysis. A.B. and D.J.E. prepared the manuscript. All authors discussed the results and commented on the manuscript.

\section{Additional information}

Accession codes: iCLIP and RNA-seq data available via the Gene Expression Omnibus (GEO) using GEO accession number GSE59335.

Supplementary Information accompanies this paper at http://www.nature.com/ naturecommunications

Competing financial interests: The authors declare no competing financial interests. 
Reprints and permission information is available online at http://npg.nature.com/ reprintsandpermissions/

How to cite this article: Best, A. et al. Human Tra2 proteins jointly control a CHEK1 splicing switch amongst alternative and constitutive target exons. Nat. Commun. 5:4760 doi: $10.1038 /$ ncomms5760 (2014). (c) (i) This work is licensed under a Creative Commons Attribution 4.0 International License. The images or other third party material in this article are included in the article's Creative Commons license, unless indicated otherwise in the credit line; if the material is not included under the Creative Commons license, users will need to obtain permission from the license holder to reproduce the material. To view a copy of this license, visit http://creativecommons.org/licenses/by/4.0/ 